\title{
Multifrequency Excitation of an Inclined Marine Riser under Internal Resonances
}

\author{
Feras K. Alfosail \\ Mohammad I. Younis* \\ Physical Sciences and Engineering Division, King Abdullah University of Science \\ and Technology, Thuwal 23955-9600, Saudi Arabia.
}

\begin{abstract}
We study the multifrequency excitation of an inclined marine riser under two-to-one and three-toone internal resonances. The riser model accounts for the initial static deflection, self-weight, and mid-plane stretching nonlinearity. By tuning the initial applied tension and configuration angles of the riser, the ratio between its first and third natural frequencies approaches two. In another case, the ratio between its first and fifth natural frequencies approaches three. As recently revealed by experimental observations, a riser can experience multifrequency vortex-induced vibrations. Hence here, the excitation frequencies are tuned such that one frequency is near the first primary resonance while the other frequency is near the second primary resonance. The multiple time scales perturbation method is used to analyze the nonlinear motion of the riser considering the internal resonances. Frequency response results of the perturbation method are compared to a Galerkin solution, which show good agreement. The perturbation results in the two-to-one internal resonance case demonstrate that increasing the forcing amplitude at the second primary resonance suppresses the energy exchange due to internal resonance and reduces the likelihood of Hopf bifurcations while an opposite trend is observed in the three-to-one internal resonance case. Then, the dynamic solutions of the modulation equations of the perturbation method are analyzed using the Floquet theory to examine the post-Hopf bifurcation response. The limit cycle responses in both internal resonance cases exhibit several period doubling bifurcations possibly leading to quasi-periodic and other complex motions, which can endanger the life of the riser.
\end{abstract}

Keywords: two-to-one internal resonance, three-to-one internal resonance, multifrequency excitation, marine riser, vortex-induced vibrations.

\footnotetext{
${ }^{*}$ Corresponding author: Mohammad I. Younis $(\bowtie)$, Physical Sciences and Engineering Division, King Abdullah University of Science and Technology, Thuwal 23955-9600, Saudi Arabia, e-mail: Mohammad.Younis@kaust.edu.sa, tel.:+966544700154, ORCID: orcid.org/0000-0002-9491-1838
} 


\section{Introduction}

Marine risers are subjected to various environmental loads, such as vortexinduced vibration (VIV) that can hinder the life of the riser by fatigue [1]. The presence of nonlinear geometrical features with the initial static deflection of the riser induces quadratic and cubic nonlinearities resulting into the possibility of activating internal resonances. During internal resonance, energy is transferred from higher frequency to lower frequency modes and vise-versa, which is considered harmful to the riser. Furthermore, the study of VIV is very complex involving interactions of different harmonics with the motion of the riser structure. Experimental observations [2-7] of VIV phenomena of vertical, inclined and curved risers demonstrate that under certain circumstances the riser can experience excitations from higher order harmonics due to the fluid interaction with the riser resulting in multifrequency vortex-induced vibrations.

Investigating multifrequency excitation of structures is essential when the structure is exposed to environmental loads consisting of multiple harmonics in a continuous system such as the fluid-structure interaction problems in risers. Several early works considered exciting nonlinear systems having quadratic and cubic nonlinearities with multifrequency excitation including the work of Nayfeh $[8,9]$ who showed that exciting a system with secondary excitations of the superharmonic and combination type along with the primary resonance under certain conditions can result in sever reductions "quenching" or enhancement of the response. Plaut et al. [10] extended the work of Nayfeh by considering two frequency excitations for a system under two-to-one internal resonance. They observed that the type of the secondary excitation term has a major role in controlling the saturation phenomenon. In the absence of internal resonance, Plaut and Hsieh [11] studied two-frequency excitations in shallow arches. They found 
that the secondary excitation causes the system to exceed critical loads and hence chaotic motions are observed. Pezeshki et al. [12] while studying multifrequency excitations of buckled beam showed that chaotic behavior can be reduced by controlling the phase of the secondary excitation term. Luongo and Piccardo [13] considered a three order expansion of the galloping forces to study the two-to-one internal resonance in sagged cables. More recently, Zhao et al. [14,15] investigated the influence of two-frequency excitations of suspended cables considering the influence of temperature effects in the absence of internal resonances. More examples on multifrequency excitations of nonlinear systems can be found in [16].

The numerical analysis of vortex induced vibration forces on risers requires computational fluid dynamics (CFD) tools to analyze the hydrodynamic forces on the riser structure. Alternatively, one can utilize semi-empirical formulations that describe some VIV features, such as the cross-flow vibration by Khalak and Williamson [17], Balasubramanian et al. [18], and Facchinetti et al. [19] considering the multifrequency nature of the cross-flow, i.e., odd harmonics, using a Van Der Pol oscillator. From that perspective and considering static deflection of the riser, Mazzilli and Sacnhez [20] analyzed the VIV of a catenary riser using the method of nonlinear normal modes to reduce the complexity of the finite element FE formulation. Srinil [21] and Srinil et al. [22] considered the twoto-one internal resonance of a catenary riser accounting for the influence of bending and used the Galerkin method to project the VIV motions along the linear modes. The results showed that there is an energy transfer among the participating modes that will interact with the VIV motion due to the activation of internal resonance. The work of Meng and Chen [23] considered the influence of the internal fluid flow of a catenary riser under the influence of VIV forces and solved 
the problem using the FE method. Dai et al. [24] numerically examined the influence of the internal fluid velocity of a post-buckled pipe subjected to VIV forces.

Despite all the extensive research work to analyze the VIV motions of risers, internal resonances in riser structures are rarely considered. In addition, risers are subjected to both cross-flow and in-line forces where the latter causes even harmonics to appear $[25,26]$ in the response during VIV. A comprehensive study describing and quantifying such forces with structural nonlinearities considering the influence of internal resonances has not yet been presented. In addition, generic external flows can be at an incident angle, which is not orthogonal to the plane of curvature causing multiple harmonics to participate in the VIV phenomena. In such cases, it is crucial to understand what happens to the motion of the structure if these harmonics interact while internal resonances are present.

In an earlier work [27,28], we studied the two-to-one and three-to-one internal resonances of inclined marine risers when the excitation is near the first primary resonance, which is a first-order approximation describing the drag and lift forces based on the analysis of the CFD results in [29]. Here, the objective is to study the effect of the vortex shedding forces by including higher order harmonics that affect the two-to-one and three-to-one internal resonances. Thus, we extend the work and consider the second-order correction, which include higher order harmonics of the drag and lift forces based on the analysis in [30] and [31] resulting into a multifrequency excitation of the riser through the first and second primary resonances of the modes participating in internal resonance. The presence of internal resonances along with the simultaneous forcing terms induced by the vortex shedding forces makes the response and behavior of the riser structure different from that observed in previous studies under harmonic 
excitation. In this way, the riser is subjected to simultaneous primary excitations of the two involved modes and internal resonances. The organization of the paper is as follows. In Sec. 2, we present the problem formulation followed by perturbation analysis in Sec. 3 considering two-to-one and three-to-one internal resonances. Then, the numerical results and discussions are presented in Sec. 4 for each internal resonance case followed by conclusions in Sec. 5 .

\section{Problem Formulation}

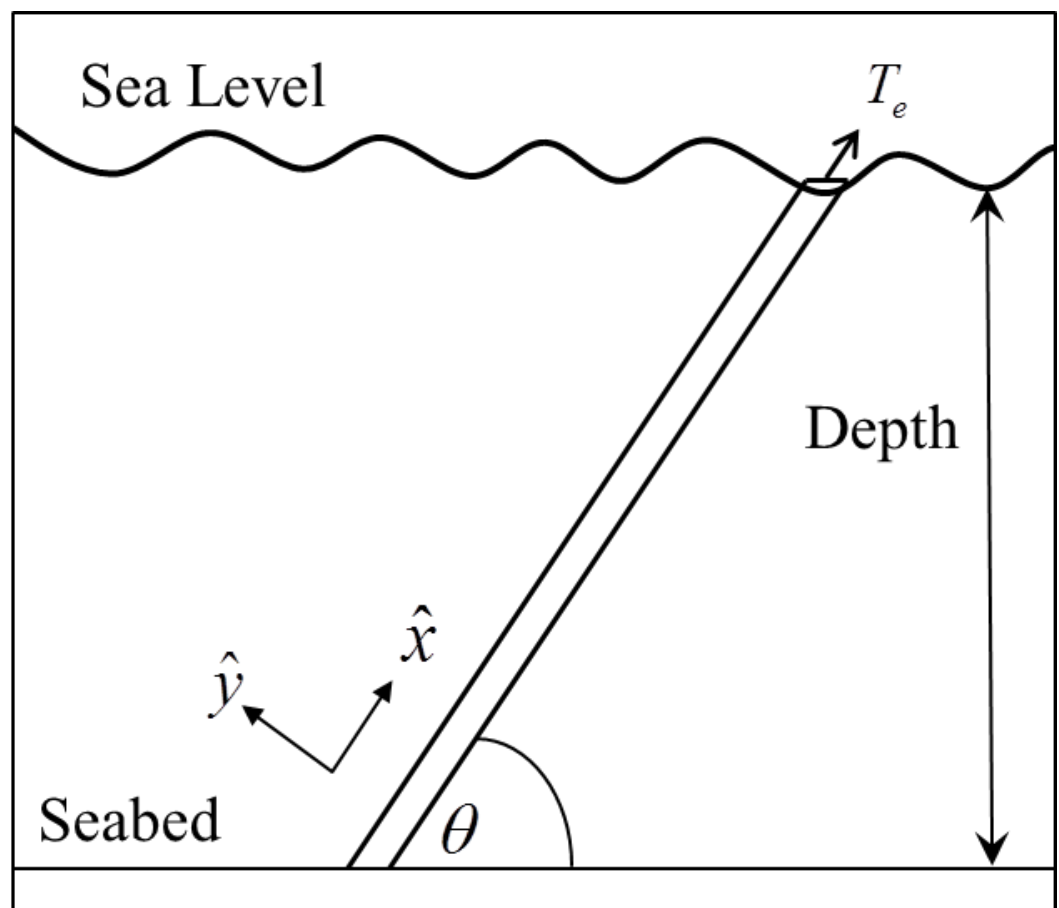

Figure 1: Schematic of the inclined riser used in the analysis.

The riser shown in Fig. 1 is modeled as an Euler-Bernoulli beam with a variable axial tension including the nonlinear geometric effects induced by mid-plane stretching. The riser is also subjected to environmental loads due to the influence of drag and lift forces inducing vortex induced vibrations. Then, the riser equation that governs the deflection $\hat{y}$ at position $\hat{x}$ and time $\hat{t}$ is given by 


$$
\begin{aligned}
& m \frac{\partial^{2} \hat{y}}{\partial \hat{t}^{2}}+E I \frac{\partial^{4} \hat{y}}{\partial \hat{x}^{4}}-\left(T_{e}-W_{e} \sin (\theta)(L-\hat{x})+\frac{E A_{p}}{2 L} \int_{0}^{L}\left(\frac{\partial \hat{y}}{\partial \hat{x}}\right)^{2} d \hat{x}\right)\left(\frac{\partial^{2} \hat{y}}{\partial \hat{x}^{2}}\right) \\
& -W_{e} \sin (\theta)\left(\frac{\partial \hat{y}}{\partial \hat{x}}\right)+\bar{c} \frac{\partial \hat{y}_{d}}{\partial \hat{t}}=\bar{F}_{d}-W_{e} \cos (\theta)
\end{aligned}
$$

where $m$ is the total mass of the riser pipe per unit length including the fluid added mass, $E I$ is the flexural rigidity, $T_{e}$ is the applied tension, $W_{e}$ is the apparent weight defined by $W_{e}=\left(m-\rho_{e} A_{e}\right) g, \rho_{e}$ is the density of sea water, $A_{e}$ is the area of the riser pipe, $g$ is the gravitational constant, $\theta$ is the inclination angle, $L$ is the length of the pipe, $A_{p}$ is the cross section area of the pipe, $\bar{c}$ is the structural damping, and $\bar{F}_{d}$ is the dynamic applied load. The axial load is assumed to vary linearly with the weight of the pipe. Our aim is to study the nonlinear vibrations of the riser around its static configuration. Therefore, the solution consists of a static component $\hat{y}_{s}$ and a dynamic component $\hat{y}_{d}$ :

$$
\hat{y}(\hat{x}, t)=\hat{y}_{s}(\hat{x})+\hat{y}_{d}(\hat{x}, t)
$$

The influence of the static loading from drag forces is neglected in the analysis for simplicity. Then, the static differential equation of the inclined riser is obtained by dropping the time dependent terms in Eq. (1), which yields

$$
\begin{aligned}
& E I \frac{d^{4} \hat{y}_{s}}{d \hat{x}^{4}}-\left(T_{e}-W_{e} \sin (\theta)(L-\hat{x})+\frac{E A_{p}}{2 L} \int_{0}^{L}\left(\frac{d \hat{y}_{s}}{d \hat{x}}\right)^{2} d \hat{x}\right)\left(\frac{d^{2} \hat{y}_{s}}{d \hat{x}^{2}}\right) \\
& -W_{e} \sin (\theta)\left(\frac{d \hat{y}_{s}}{d \hat{x}}\right)=-W_{e} \cos (\theta)
\end{aligned}
$$

The static equation is solved using the method of matched asymptotic expansion [32]. Then, the dynamic equation of the inclined riser around equilibrium is obtained by substituting Eq. (2) into Eq. (1) and dropping the terms from Eq. (3), which yields 


$$
\begin{aligned}
& m \frac{\partial^{2} \hat{y}_{d}}{\partial \hat{t}^{2}}+E I \frac{\partial^{4} \hat{y}_{d}}{\partial \hat{x}^{4}}-\left(T_{e}-W_{e} \sin (\theta)(L-\hat{x})\right)\left(\frac{\partial^{2} \hat{y}_{d}}{\partial \hat{x}^{2}}\right) \\
& -\left(\frac{E A_{p}}{2 L}\left(\int_{0}^{L}\left(\frac{d \hat{y}_{s}}{d \hat{x}}\right)^{2} d \hat{x}+\int_{0}^{L}\left(\frac{\partial \hat{y}_{d}}{\partial \hat{x}}\right)^{2} d \hat{x}+\int_{0}^{L}\left[2 \frac{d \hat{y}_{s}}{d \hat{x}} \frac{\partial \hat{y}_{d}}{\partial \hat{x}}\right] d \hat{x}\right)\right)\left(\frac{\partial^{2} \hat{y}_{d}}{\partial \hat{x}^{2}}\right) \\
& -\left(\frac{E A_{p}}{2 L}\left(\int_{0}^{L}\left[2 \frac{d \hat{y}_{s}}{d \hat{x}} \frac{\partial \hat{y}_{d}}{\partial \hat{x}}\right] d \hat{x}+\int_{0}^{L}\left(\frac{\partial \hat{y}_{d}}{\partial \hat{x}}\right)^{2} d \hat{x}\right)\right)\left(\frac{d^{2} \hat{y}_{s}}{d \hat{x}^{2}}\right) \\
& -W_{e} \sin (\theta)\left(\frac{\partial \hat{y}_{d}}{\partial \hat{x}}\right)+\bar{c} \frac{\partial \hat{y}_{d}}{\partial \hat{t}}=\bar{F}_{d}
\end{aligned}
$$

To simplify the analysis, we introduce the following dimensionless variables:

$$
y=\frac{\hat{y}}{D} ; \quad x=\frac{\hat{x}}{L} ; \quad t=\sqrt{\frac{E I}{m L^{4}}} \hat{t}
$$

where the "hat" denotes the dimensional variables and the variables without the "hat" are dimensionless. Substituting Eq. (5) into (4) yields

$$
\begin{aligned}
& \frac{\partial^{2} y_{d}}{\partial t^{2}}+\frac{\partial^{4} y_{d}}{\partial x^{4}}-\left(\bar{T}-\bar{\sigma}(1-x)-\bar{\eta}\left(\int_{0}^{1}\left(\frac{d y_{s}}{d x}\right)^{2} d x+\int_{0}^{1}\left(\frac{\partial y_{d}}{\partial x}\right)^{2} d x+\int_{0}^{1} 2 \frac{d y_{s}}{d x} \frac{\partial y_{d}}{\partial x} d x\right)\right)\left(\frac{\partial^{2} y_{d}}{\partial x^{2}}\right) \\
& -\left(\bar{\eta}\left(\int_{0}^{1} 2 \frac{d y_{s}}{d x} \frac{\partial y_{d}}{\partial x} d x+\int_{0}^{1}\left(\frac{\partial y_{d}}{\partial x}\right)^{2} d \hat{x}\right)\right)\left(\frac{d^{2} y_{s}}{d x^{2}}\right)-\bar{\sigma}\left(\frac{\partial y_{d}}{\partial x}\right)+c \frac{\partial y_{d}}{\partial t}=F_{d}
\end{aligned}
$$

where

$$
\begin{aligned}
& \bar{\sigma}=\frac{W_{e} \sin (\theta) L^{3}}{E I} ; \quad \bar{\eta}=\frac{A_{p} D^{2}}{2 I} ; \quad c=\frac{\bar{c} L^{2}}{\sqrt{m E I}} ; \\
& F_{d}=\frac{L^{4} \bar{F}_{d}}{E I D} ; \quad \bar{T}=\frac{T_{e} L^{2}}{E I}
\end{aligned}
$$

From Eq. (6), we note the presence of quadratic and cubic nonlinearities, which arise due to the stretch and initial static deflection of the riser structure, in addition to a linear term that affects the eigenvalue problem. Next, to obtain the natural frequencies and mode shapes of the riser, the linear eigenvalue problem is analyzed by examining the linearized form of Eq. (6) after dropping damping, forcing and the nonlinear terms, which yields 


$$
\frac{\partial^{2} y_{d}}{\partial t^{2}}+\frac{\partial^{4} y_{d}}{\partial x^{4}}+\left(\overline{T_{s}}-\bar{\sigma} x-\bar{\eta} \int_{0}^{1}\left(\frac{d y_{s}}{d x}\right)^{2} d x\right) \frac{\partial^{2} y_{d}}{\partial x^{2}}-\bar{\eta}\left(\int_{0}^{1} 2 \frac{d y_{s}}{d x} \frac{\partial y_{d}}{\partial x} d x\right)\left(\frac{d^{2} y_{s}}{d x^{2}}\right)-\bar{\sigma} \frac{\partial y_{d}}{\partial x}=0
$$

where $\bar{T}_{s}=\bar{\sigma}-\bar{T}$. The mode shapes and the natural frequencies of the structure are extracted by solving Eq. (8) using the Galerkin procedure [33]. The next step is to solve the forced vibration problem. For that, the description of the forcing term $F_{d}$ needs to be addressed. Often, computation fluid dynamics is the approach to solve VIV problem. However, the numerical computations involved while considering structural interactions make the use of CFD tools computationally expensive. Alternatively, simplified lift and drag models have been proposed such as the Van der pol and Morison formulation. In this context, we consider the projection of the drag and lift forces on the riser structure and neglect the influence of coupling and synchronization to simplify the analysis of the internal resonance interaction [34]. The work presented by Marzouk et al. [30] and Dahl et al. [31] showed that the the drag and lift forces in VIV can be described by

$$
\begin{aligned}
& F_{\text {lift }}=f_{\Omega_{s}} \cos \left(\Omega_{s} t\right)+f_{3 \Omega_{s}} \cos \left(3 \Omega_{s} t\right) \\
& F_{\text {drag }}=f_{2 \Omega_{s}} \cos \left(2 \Omega_{s} t\right)
\end{aligned}
$$

where $f_{i \Omega_{s}}$ is the forcing amplitude and $\Omega_{s}$ is the vortex shedding excitation frequency. For simplicity, the influence of phases from each frequency and the static term of drag are neglected. Then, the total dynamic force acting on the riser is given by

$$
F_{d}=F_{\text {drag }}+F_{\text {lift }}=f_{\Omega_{s}} \cos \left(\Omega_{s} t\right)+f_{2 \Omega_{s}} \cos \left(2 \Omega_{s} t\right)+f_{3 \Omega_{s}} \cos \left(3 \Omega_{s} t\right)
$$

The selection of the dynamic force in Eq. (10) depends on several limiting factors: First, the VIV characteristics in which both $2 \Omega$ and $3 \Omega$ excitation terms exist can only be obtained when the external current flow is neither orthogonal (cross-flow) nor inline (in-flow) with the plane containing the curvature of the riser. This can 
be only solved numerically and it will require three dimensional model of the structure coupled with CFD solvers which are quiet expensive. Second, the lift force induced by the VIV phenomena from the cross-flow can be described by a semi-empirical formulation via a Van der Pol oscillator synchronized with the motion of the riser. In such a case, the influence of the $2 \Omega$ force term is not captured and only the influence of the odd harmonics is captured. Also, the influence of the phase and amplitude scaling are neglected for the purpose of studying the extreme forcing limits on the response of the riser under internal resonances considering the worst case scenario. Therefore, the influence of the excitation terms in Eq. (10) becomes pronounced in the presence of the internal resonance. In the next section, a perturbation analysis using multiple time scales is carried out on Eq. (6) considering the two-to-one and three-to-one internal resonances.

\section{Perturbation Analysis}

\subsection{Two-To-One Internal Resonance}

In this section, we use the method of multiple time scales [35] to analyze the response of the structure under two-to-one internal resonance and consider multifrequency excitations. We seek a three-order expansion of the form

$$
y_{d}(x, t ; \varepsilon)=\varepsilon y_{1}\left(x, T_{0}, T_{1}, T_{2}\right)+\varepsilon^{2} y_{2}\left(x, T_{0}, T_{1}, T_{2}\right)+\varepsilon^{3} y_{3}\left(x, T_{0}, T_{1}, T_{2}\right)
$$

where $\varepsilon$ is introduced as a bookkeeping parameter, and $T_{0}=\varepsilon t, T_{1}=\varepsilon t, T_{2}=\varepsilon^{2} t$. Thus, the auto scaling chosen at Eq. (11) determines that the quadratic nonlinearity is at $\varepsilon^{2}$ and the cubic nonlinearity is at $\varepsilon^{3}$ while the forcing terms are assumed to be $\varepsilon^{2} f_{\Omega_{s}} \cos \left(\Omega_{s} t\right), \varepsilon^{2} f_{2 \Omega_{s}} \cos \left(2 \Omega_{s} t\right)$ and damping is assumed to be 
$\varepsilon c$. In this case, the influence of the $3 \Omega_{s}$ term is neglected from the analysis (will be considered in the three-one resonance case). We substitute Eq. (11) and Eq. (10) into Eq. (6) with the appropriate scales to obtain

$$
\begin{gathered}
\varepsilon^{1}: \ell\left(y_{1}\right)=D_{0}^{2} y_{1}+y_{1}^{i v}+\bar{T}_{s} y_{1}^{\prime \prime}-x \bar{\sigma} y_{1}^{\prime \prime}-\bar{\sigma} y_{1}^{\prime} \\
-2 \eta y_{s}^{\prime \prime}(x)\left(\int_{0}^{1} y_{s}^{\prime}(x) y_{1}^{\prime} \mathrm{d} x\right)=0 \\
\varepsilon^{2}: \ell\left(y_{2}\right)=-c D_{0} y_{1}-2 D_{0} D_{1} y_{1}+\eta y_{s}^{\prime \prime}(x)\left(\int_{0}^{1}\left(y_{1}^{\prime}\right)^{2} \mathrm{~d} x\right) \\
+2 \eta y_{1}^{\prime \prime}\left(\int_{0}^{1} y_{s}^{\prime}(x) y_{1}^{\prime} \mathrm{d} x\right)+\frac{f_{\Omega_{s}}}{2} e^{i_{\Omega_{s}} T_{0}}+\frac{f_{2 \Omega_{s}}}{2} e^{i 2 \Omega_{s} T_{0}} \\
\varepsilon^{3}: \ell\left(y_{3}\right)=-D^{2} y_{1}-2 D_{0} D_{2} y_{1}-2 D_{0} D_{1} y_{2}-c D_{1} y_{1}-c D_{0} y_{2} \\
+2 \eta\left(y_{2}^{\prime \prime} \int_{0}^{1} y_{s}^{\prime} y_{1}^{\prime} \mathrm{d} x+y_{s}^{\prime \prime} \int_{0}^{1} y_{1}^{\prime} y_{2}^{\prime} \mathrm{d} x+y_{1}^{\prime \prime} \int_{0}^{1} y_{s}^{\prime} y_{2}^{\prime} \mathrm{d} x\right)+\eta y_{1}^{\prime \prime} \int_{0}^{1}\left(y_{1}^{\prime}\right)^{2} \mathrm{~d} x
\end{gathered}
$$

where $\ell(\quad)$ defines the linear operator given in Eq. (12), the derivatives are defined as $D_{i}^{j}=\frac{\partial}{\partial T_{i}^{j}}$ and ( )' denotes the spatial derivative with respect to $x$. From Eq. (13), we observe the participation of both excitation terms in the second order to contribute to the two-to-one internal resonance. Next, we follow the multiple time scales procedure to derive a solvability condition in the third order accounting for the influence of quadratic and cubic nonlinearities and assuming $\omega_{n}=2 \omega_{m}+\varepsilon^{2} \delta$ and the forcing term $\Omega_{s}=\omega_{m}+\varepsilon^{2} \alpha$, where $\omega_{n}$ and $\omega_{m}$ are the natural frequencies of modes $m$ and $n$, and $\delta$ and $\alpha$ are the frequency detuning parameters. Then, the modulation equations that describe the amplitude and phase in polar coordinates are given by 


$$
\begin{aligned}
& \dot{a}_{m}=-\frac{\mu_{1}}{2} a_{m}+\frac{\mu_{1} F_{\Omega_{s}}}{4 \omega_{m}^{2}} \cos \left(\beta_{m}\right)-\frac{F_{\Omega_{s}}}{\omega_{m}} \sin \left(\beta_{m}\right)-\frac{R_{1} F_{\Omega_{s}}}{8 \omega_{m}^{3}} a_{n} \sin \left(\beta_{m}-\beta_{n}\right) \\
& -\frac{R_{1} F_{2 \Omega_{s}}}{8 \omega_{m} \omega_{n}^{2}} a_{m} \sin \left(2 \beta_{m}\right)+\left(\frac{\mu_{1} R_{1}}{8 \omega_{m}^{2}}-\frac{\mu_{1} R_{1}}{16 \omega_{m} \omega_{n}}\right) a_{m} a_{n} \cos \left(2 \beta_{m}-\beta_{n}\right) \\
& -\frac{R_{1}}{4 \omega_{m}} a_{m} a_{n} \sin \left(2 \beta_{m}-\beta_{n}\right) \\
& a_{m} \dot{\beta}_{m}=-\left(\alpha+\frac{\mu_{1}^{2}}{8 \omega_{m}}\right) a_{m}-\frac{\mu_{1} F_{\Omega_{s}}}{4 \omega_{m}^{2}} \sin \left(\beta_{m}\right)-\frac{F_{\Omega_{s}}}{\omega_{m}} \cos \left(\beta_{m}\right) \\
& -\frac{R_{1} F_{2 \Omega_{s}}}{8 \omega_{m} \omega_{n}^{2}} a_{m} \cos \left(2 \beta_{m}\right)-\frac{R_{1} F_{\Omega_{s}}}{8 \omega_{m}^{3}} a_{n} \cos \left(\beta_{m}-\beta_{n}\right) \\
& -\left(\frac{K_{2}}{8 \omega_{m}}+\frac{R_{1} R_{2}}{32 \omega_{m} \omega_{n}^{2}}\right) a_{m}^{3}-\left(\frac{K_{1}}{8 \omega_{m}}+\frac{R_{1}^{2}}{32 \omega_{m}^{3}}\right) a_{m} a_{n}^{2} \\
& +\left(\frac{\mu_{1} R_{1}}{16 \omega_{m} \omega_{n}}-\frac{\mu_{1} R_{1}}{8 \omega_{m}^{2}}\right) a_{m} a_{n} \sin \left(2 \beta_{m}-\beta_{n}\right)-\frac{R_{1}}{4 \omega_{m}} a_{m} a_{n} \cos \left(2 \beta_{m}-\beta_{n}\right) \\
& \dot{a}_{n}=-\frac{\mu_{1}}{2} a_{n}+\frac{\mu_{1} F_{2 \Omega_{s}}}{4 \omega_{n}^{2}} \cos \left(\beta_{n}\right)-\frac{F_{2 \Omega_{s}}}{\omega_{n}} \sin \left(\beta_{n}\right) \\
& +\frac{R_{2} F_{\Omega_{s}}}{4 \omega_{m}^{2} \omega_{n}} a_{m} \sin \left(\beta_{m}-\beta_{n}\right)+\left(\frac{\mu_{1} R_{2}}{16 \omega_{n}^{2}}-\frac{\mu_{1} R_{2}}{8 \omega_{m} \omega_{n}}\right) a_{m}^{2} \cos \left(2 \beta_{m}-\beta_{n}\right) \\
& +\frac{R_{2}}{4 \omega_{n}} a_{m}^{2} \sin \left(2 \beta_{m}-\beta_{n}\right) \\
& a_{n} \dot{\beta}_{n}=\left(\delta-2 \alpha-\frac{\mu_{1}^{2}}{8 \omega_{n}}\right) a_{n}-\frac{\mu_{1} F_{2 \Omega_{s}}}{4 \omega_{n}^{2}} \sin \left(\beta_{n}\right)-\frac{F_{2 \Omega_{s}}}{\omega_{n}} \cos \left(\beta_{n}\right) \\
& -\frac{R_{2} F_{\Omega_{s}}}{4 \omega_{m}^{2} \omega_{n}} a_{m} \cos \left(\beta_{m}-\beta_{n}\right)-\left(\frac{K_{3}}{8 \omega_{n}}+\frac{R_{1} R_{2}}{16 \omega_{m}^{2} \omega_{n}}\right) a_{m}^{2} a_{n}-\frac{K_{4}}{8 \omega_{n}} a_{n}^{3} \\
& +\left(\frac{\mu_{1} R_{2}}{16 \omega_{n}^{2}}-\frac{\mu_{1} R_{2}}{8 \omega_{m} \omega_{n}}\right) a_{m}^{2} \sin \left(2 \beta_{m}-\beta_{n}\right)-\frac{R_{2}}{4 \omega_{n}} a_{m}^{2} \cos \left(2 \beta_{m}-\beta_{n}\right)
\end{aligned}
$$

where the dot denotes the derivative with respect to time, $a_{i}$ are the amplitudes, $\beta_{i}$ are the phases, $R_{1}, R_{2}, K_{1}, K_{2}, K_{3}, K_{4}$ and $\mu_{1}$ are defined in Appendix A, and $F_{i \Omega_{s}}$ represents the projected modal force. The modulation equations can be written in complex Cartesian form to study the stability of the response, which is found in Appendix B. The final solution considering the interaction is written as 


$$
\begin{aligned}
y_{d}(x, t)= & a_{m} \phi_{m}(x) \cos \left(\Omega_{s} t+\beta_{m}\right)+a_{n} \phi_{n}(x) \cos \left(2 \Omega_{s} t+\beta_{n}\right)+ \\
& a_{m} \frac{\mu_{1}}{4 \omega_{m}} \phi_{m}(x) \sin \left(\Omega_{s} t+\beta_{m}\right)+a_{n} \frac{\mu_{1}}{4 \omega_{n}} \phi_{n}(x) \sin \left(2 \Omega_{s} t+\beta_{n}\right)+ \\
& +\frac{R_{2}}{8 \omega_{n}^{2}} a_{m}^{2} \phi_{n}(x) \cos \left(2 \Omega_{s} t+2 \beta_{m}\right) \\
& +\frac{R_{1}}{8 \omega_{m}^{2}} a_{m} a_{n} \phi_{m}(x) \cos \left(\Omega_{s} t+\beta_{n}-\beta_{m}\right) \\
& +\frac{1}{2} a_{m} a_{n} \psi_{1}(x) \cos \left(3 \Omega_{s} t+\beta_{m}+\beta_{n}\right) \\
& +\frac{1}{2} a_{n}^{2} \psi_{2}(x) \cos \left(4 \Omega_{s} t+2 \beta_{n}\right)+\frac{1}{4} a_{m}^{2} \psi_{3}(x)+\frac{1}{4} a_{n}^{2} \psi_{4}(x) \\
& +\frac{F_{\Omega_{s}}}{2 \omega_{m}^{2}} \phi_{m}(x) \cos \left(\Omega_{s} t\right)+\frac{F_{2 \Omega_{s}}}{2 \omega_{n}^{2}} \phi_{n}(x) \cos \left(2 \Omega_{s} t\right)
\end{aligned}
$$

where $\phi_{i}(x)$ are the mode shapes and $\psi_{i}(x)$ are the particular solution obtained in the multiple time scales procedure.

\subsection{Three-To-One Internal Resonance}

Here, the forcing and damping scales are chosen such that they appear at the third order, i.e., $\varepsilon^{3} f_{\Omega_{s}} \cos \left(\Omega_{s} t\right), \varepsilon^{3} f_{3 \Omega_{s}} \cos \left(3 \Omega_{s} t\right)$ and $\varepsilon^{2} c$, respectively. Then, we substitute into Eq. (10) and Eq. (11) to obtain

$$
\begin{aligned}
\varepsilon^{1}: \ell\left(y_{1}\right)= & D_{0}^{2} y_{1}+y_{1}^{i v}+\bar{T}_{s} y_{1}^{\prime \prime}-x \bar{\sigma} y_{1}^{\prime \prime}-\bar{\sigma} y_{1}^{\prime}-2 \eta y_{s}^{\prime \prime}(x)\left(\int_{0}^{1} y_{s}^{\prime}(x) y_{1}^{\prime} \mathrm{d} x\right)=0 \\
\varepsilon^{2}: \ell\left(y_{2}\right)= & -2 D_{0} D_{1} y_{1}+\eta y_{s}^{\prime \prime}(x)\left(\int_{0}^{1}\left(y_{1}^{\prime}\right)^{2} \mathrm{~d} x\right)+2 \eta y_{1}^{\prime \prime}\left(\int_{0}^{1} y_{s}^{\prime}(x) y_{1}^{\prime} \mathrm{d} x\right) \\
\varepsilon^{3}: & \ell\left(y_{3}\right)=-2 D_{0} D_{1} y_{2}-2 D_{0} D_{2} y_{1}-D_{1}^{2} y_{1}+\eta y_{1}^{\prime \prime}\left(\int_{0}^{1}\left(y_{1}^{\prime}\right)^{2} \mathrm{~d} x\right)+\frac{f_{\Omega_{s}}}{2} e^{i \Omega_{s} T_{0}} \\
& +\frac{f_{3 \Omega_{s}}}{2} e^{i 3 \Omega_{s} T_{0}}+2 \eta y_{s}^{\prime \prime}\left(\int_{0}^{1} y_{1}^{\prime} y_{2}^{\prime} \mathrm{d} x\right)+2 \eta y_{2}^{\prime \prime}\left(\int_{0}^{1} y_{s}^{\prime}(x) y_{1}^{\prime} \mathrm{d} x\right) \\
& +2 \eta y_{1}^{\prime \prime}\left(\int_{0}^{1} y_{s}^{\prime}(x) y_{2}^{\prime} \mathrm{d} x\right)-c D_{0} y_{1}
\end{aligned}
$$

The influence of the $2 \Omega_{s}$ excitation is neglected from this analysis because it does not directly affect the internal resonance interaction and, thus, has weak 
contribution. Following the multiple time scales procedure of the three-to-one internal resonance in [28] and considering the interaction given by $\omega_{n}=3 \omega_{m}+\varepsilon^{2} \delta$ and the forcing term $\Omega_{s}=\omega_{m}+\varepsilon^{2} \alpha$, the obtained modulation equations are

$$
\begin{aligned}
& \omega_{m} \dot{a}_{m}=-\bar{F}_{\Omega_{s}} \sin \left(\beta_{m}\right)-\mu_{1} \omega_{m} a_{m}-R_{3} a_{m}^{2} a_{n} \sin \left(3 \beta_{m}-\beta_{n}\right) \\
& \omega_{m} a_{m} \dot{\beta}_{m}=-\alpha \omega_{m} a_{m}-R_{2} a_{m} a_{n}^{2}-R_{1} a_{m}^{3}-\bar{F}_{\Omega_{s}} \cos \left(\beta_{m}\right) \\
& -R_{3} a_{m}^{2} a_{n} \cos \left(3 \beta_{m}-\beta_{n}\right) \\
& \omega_{n} \dot{a}_{n}=-\mu_{2} \omega_{n} a_{n}-\bar{F}_{3 \Omega_{s}} \sin \left(\beta_{n}\right)+K_{3} a_{m}^{3} \sin \left(3 \beta_{m}-\beta_{n}\right) \\
& \omega_{n} a_{n} \dot{\beta}_{n}=(\delta-3 \alpha) a_{n} \omega_{n}-K_{2} a_{m}^{2} a_{n}-K_{1} a_{n}^{3}-\cos \left(\beta_{n}\right) \bar{F}_{3 \Omega_{s}} \\
& -K_{3} a_{m}^{3} \cos \left(3 \beta_{m}-\beta_{n}\right)
\end{aligned}
$$

where $R_{1}, R_{2}, R_{3}, K_{1}, K_{2}, K_{3}$ in Eq. (20.1) to Eq. (20.4) are defined in Appendix A. The stability of the response is investigated by studying the eigenvalues of the Jacobain of the modulation equations in the Cartesian form as in Appendix B. Then, the full solution is expressed as

$$
\begin{aligned}
y_{d}(x, t) & =a_{m} \phi_{m}(x) \cos \left(\left(\omega_{m}+\alpha\right) t-\gamma_{1}\right) \\
& +a_{n} \phi_{n}(x) \cos \left(\left(\omega_{n}+3 \alpha-\delta\right) t+\left(\gamma_{2}-3 \gamma_{1}\right)\right) \\
& +\Pi_{1 m}(x) \frac{a_{m}{ }^{2}}{2} \cos \left(2 \omega_{m} t+2\left(\alpha t-\gamma_{1}\right)\right)+\Pi_{2 m}(x) \frac{a_{m}{ }^{2}}{2} \\
& +\Pi_{1 n}(x) \frac{a_{n}^{2}}{2} \cos \left(2\left(\omega_{n}+3 \alpha-\delta\right) t+2\left(\gamma_{2}-3 \gamma_{1}\right)\right)+\Pi_{2 n}(x) \frac{a_{n}{ }^{2}}{2} \\
& +\Pi_{1 m n}(x) \frac{a_{n} a_{m}}{2} \cos \left(\left(\omega_{n}-\omega_{m}\right) t+(3 \alpha-\delta) t+\left(\gamma_{2}-3 \gamma_{1}\right)-\left(\alpha t-\gamma_{1}\right)\right) \\
& +\Pi_{2 m n}(x) \frac{a_{n} a_{m}}{2} \cos \left(\left(\omega_{n}+\omega_{m}\right) t+(3 \alpha-\delta) t+\left(\gamma_{2}-3 \gamma_{1}\right)+\left(\alpha t-\gamma_{1}\right)\right)
\end{aligned}
$$

where $\Pi_{i j k}(x)$ represents the particular solutions obtained in the multiple time scales procedure. 


\section{Numerical Results}

\subsection{Two-to-one internal resonance}

We consider as a case study the riser of properties shown in Table 1 based on the work in [23]. In addition, the configuration of the riser is set at $\theta=62^{\circ}$ and the applied tension at $\bar{T}=2 \bar{\sigma}$ such that the frequency ratio between the third and first natural frequencies is 2 .

Table 1. The parameters of the riser used in the calculations.

\begin{tabular}{ll}
\hline Parameter & Value \\
\hline Outside Diameter, $\boldsymbol{D}$ & $0.26 \mathrm{~m}$ \\
Inside Diameter, $\boldsymbol{D}_{\boldsymbol{i}}$ & $0.2 \mathrm{~m}$ \\
Elastic Modulus, $\boldsymbol{E}$ & $207 \mathrm{GPa}$ \\
Density of Riser, $\boldsymbol{\rho}_{\boldsymbol{p}}$ & $7850 \mathrm{Kg} / \mathrm{m}^{3}$ \\
Density of Sea Water, $\boldsymbol{\rho}_{\boldsymbol{e}}$ & $1025 \mathrm{Kg} / \mathrm{m}^{3}$ \\
Depth of Sea & $150 \mathrm{~m}$ \\
\hline
\end{tabular}

First, the solution of the multiple time scales is validated against a numerical solution obtained by solving Eq. (6) using the Galerkin method utilizing five linear mode shapes [33]. The result of the validation is shown in Fig. 2.

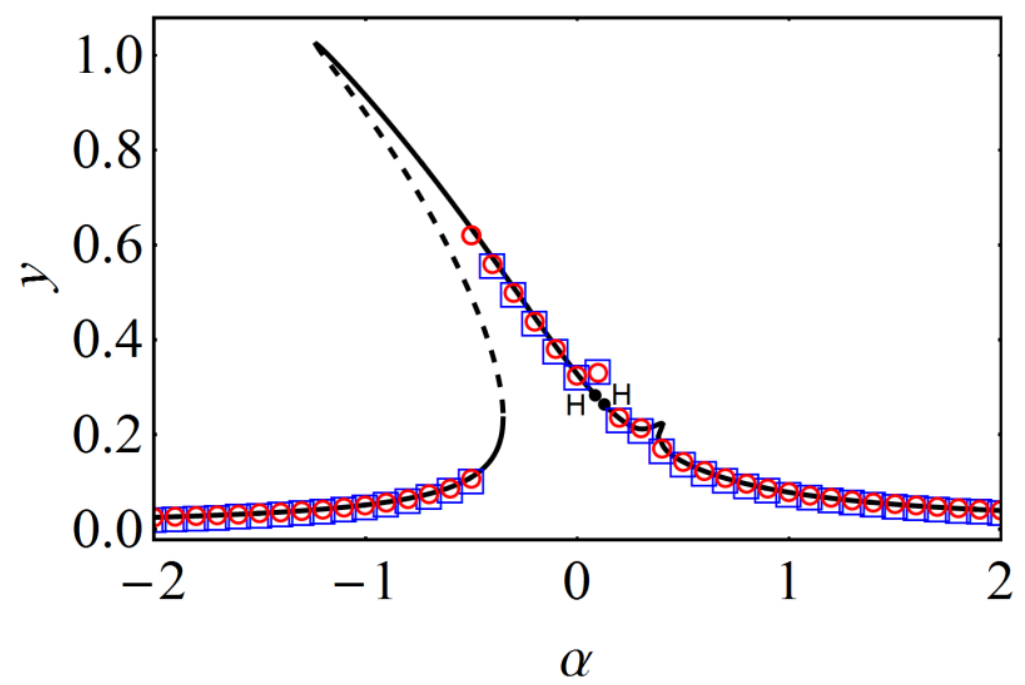

Figure 2: Frequency response curve of the riser deflection as varying the detuning parameter $\alpha$. $\theta=62^{\circ}, \mu_{1}=0.08, \mu_{3}=0.08, F_{\Omega_{s}}=8, F_{2 \Omega_{s}}=8$. ( $\square$ ) Galerkin solution, forward sweep, 
( $\bigcirc$ ) Galerkin solution, backward sweep, (-) MTS stable solution, (- - - -) MTS unstable solution. H denotes a Hopf bifurcation.

From Fig. 2, we observe good agreement between the multiple time scales solution and the one obtained by the Galerkin method. One can note that the Galerkin solution can capture the Hopf bifurcated point while cannot capture responses for $y>0.65$, even when coupled with a sequential backward continuation technique. This suggests that the basin of attraction of the Galerkin solution is very weak in that range. To study the influence of the second excitation force $F_{2 \Omega_{s}}$, we plot the amplitudes of the first and third modes at a constant $F_{\Omega_{s}}$ for different values of $F_{2 \Omega_{s}}$ in Fig. 3 and Fig. 4.

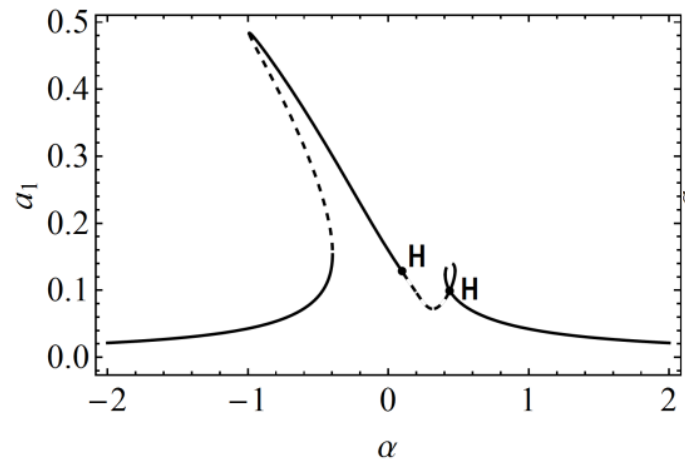

(a)

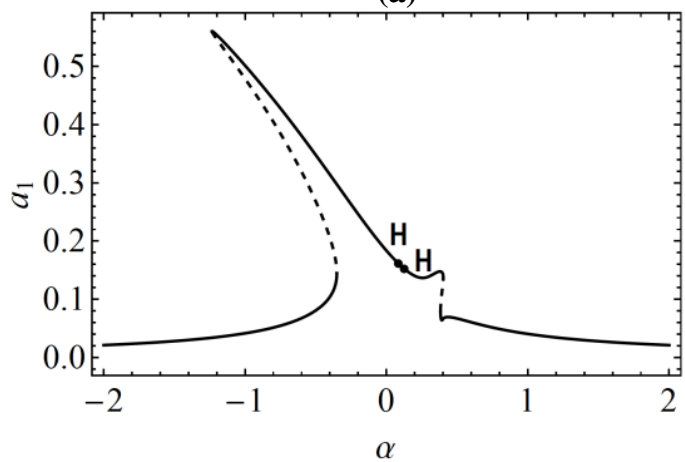

(c)

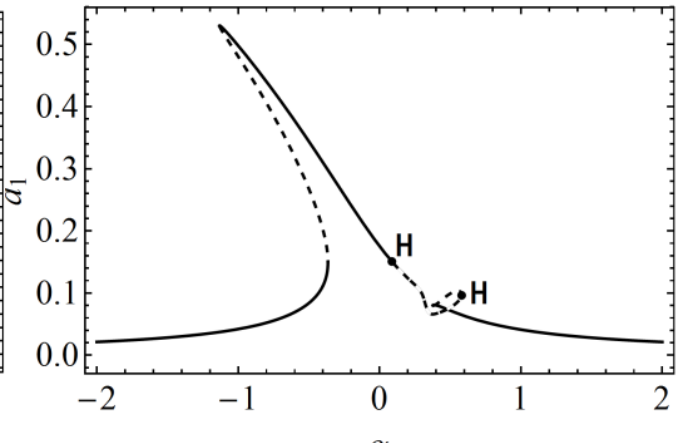

(b)

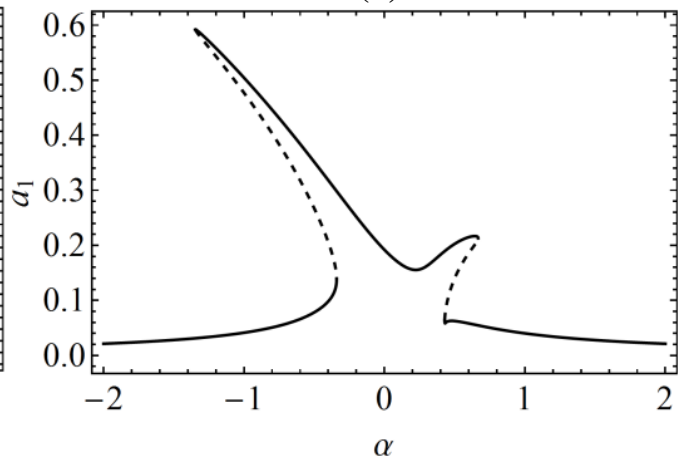

(d)

Figure 3: Frequency response curves of the first mode amplitude $a_{1}$ as varying the detuning parameter $\alpha . \theta=62^{\circ}, \mu_{1}=0.08, \mu_{2}=0.08, F_{\Omega_{s}}=8$. (a) $F_{2 \Omega_{s}}=2$, (b) $F_{2 \Omega_{s}}=6$, (c) $F_{2 \Omega_{s}}=8$, (d) $F_{2 \Omega_{s}}=10$. (• ) H denotes Hopf bifurcation. (-) Stable solution, (- - -) unstable solution. 


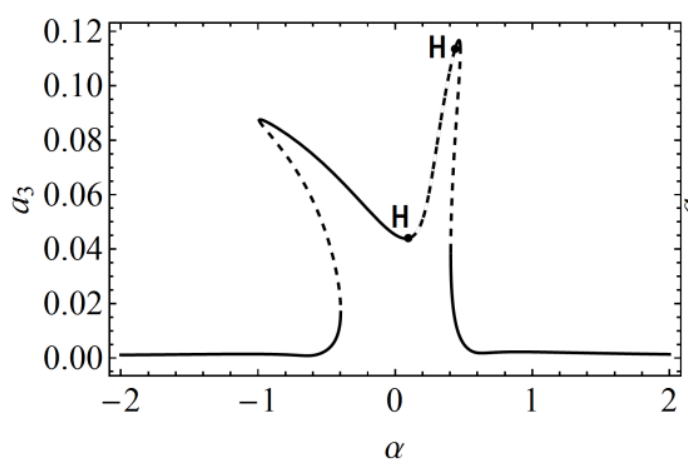

(a)

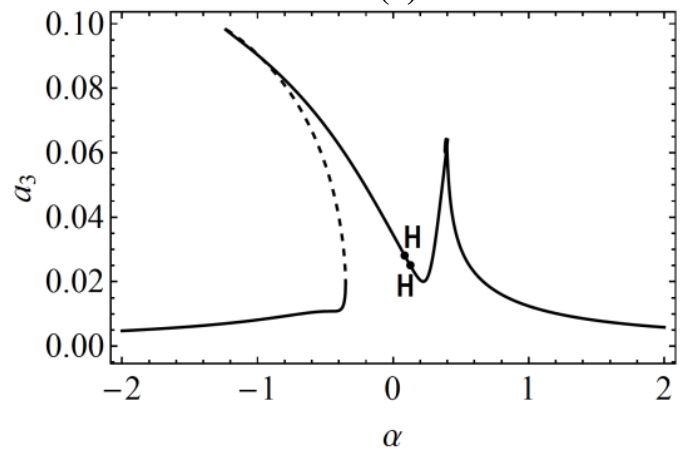

(c)

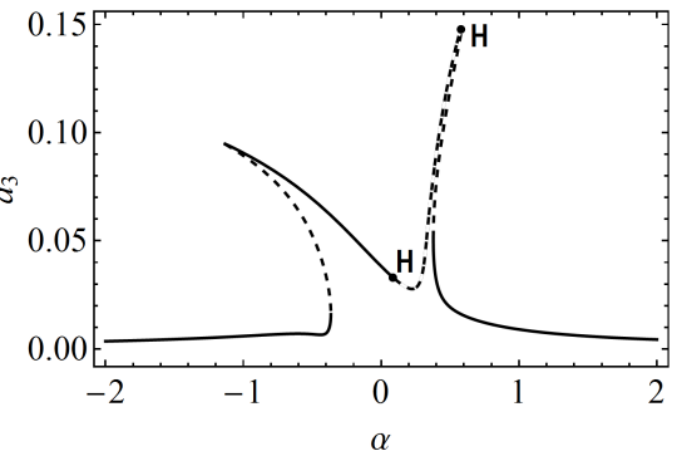

(b)

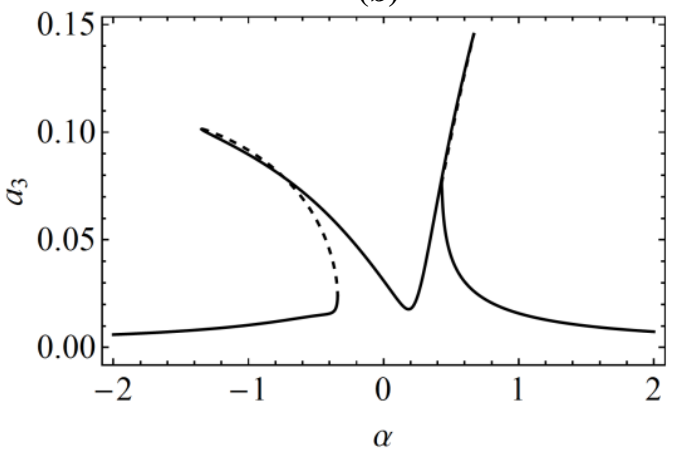

(d)

Figure 4: Frequency response curves of the third mode amplitude $a_{3}$ as varying the detuning parameter $\alpha . \theta=62^{\circ}, F_{\Omega_{s}}=8, \mu_{1}=0.08, \mu_{2}=0.08$. (a) $F_{2 \Omega_{s}}=2$, (b) $F_{2 \Omega_{s}}=6$, (c) $F_{2 \Omega_{s}}=8$, (d) $F_{2 \Omega_{s}}=10$. H denotes Hopf bifurcation. (-) Stable solution, (- - -) unstable solution.

From Fig. 3 and Fig. 4, we notice the influence of the internal resonance interaction represented by the two peaks in the first and third mode amplitudes, respectively. In addition, we notice the presence of a Hopf bifurcation band that is considered detrimental to the riser based on the nature of the born limit cycles. The range of the Hopf band decreases as the second forcing amplitude $F_{2 \Omega_{s}}$ increases and it diminishes when the forcing amplitudes exceed 8 as noted in Fig. 3d and Fig. 4d. In addition, the nonlinear energy exchange while increasing the second forcing value $F_{2 \Omega_{s}}$ indicates that there is an exchange of behavior between the first and third modes where the third modes peaks, hardening, are higher in Fig. 4a,b,d while the softening is higher in Fig. 4c. This suggests that the second forcing has a role in energy distribution among the participating modes in the interaction. The total frequency response of the riser corresponding to each 
amplitude case plotted in Fig. 3 and Fig. 4 is found in Appendix C dominated by the first mode amplitude and confirm the previous results. To better understand such phenomenon, the force response curves are plotted in Fig. 5.
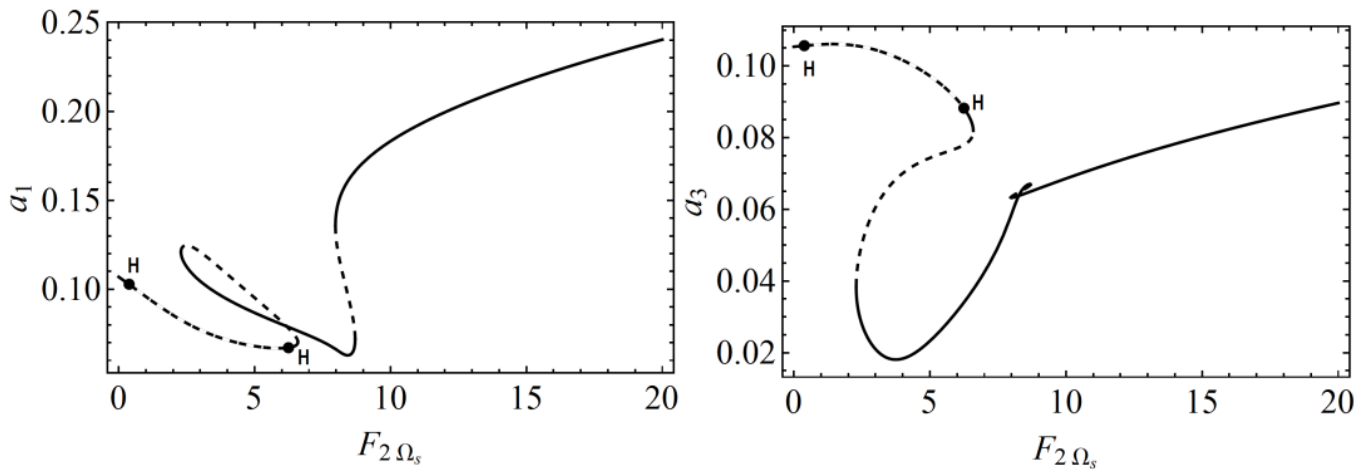

(a)

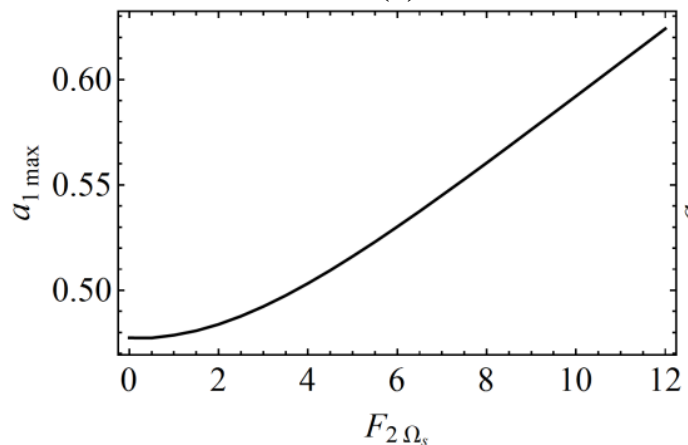

(c)

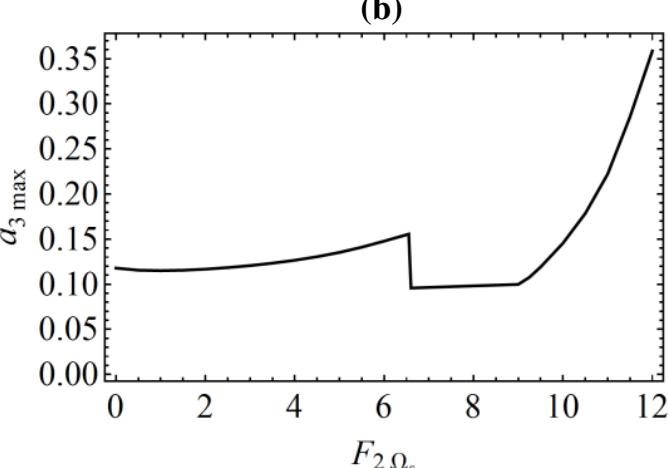

(d)

Figure 5: Force response curves for the first and third modes amplitudes as varying the force amplitude $F_{2 \Omega_{s}} . \theta=62^{\circ}, \mu_{1}=0.08, \mu_{2}=0.08, F_{\Omega_{s}}=8$. (—) Stable solution, (- -$)$ unstable solution. $(a, b) \alpha=0.40,(c, d)$ Maximum response in the frequency band. ( • ) H denotes a Hopf bifurcation.

The force response plots in Fig. 5 are obtained in two different means: (a,b) are obtained at a fixed value of frequency detuning parameter while $(\mathrm{c}, \mathrm{d})$ are obtained considering the maximum amplitude across the whole band of the frequency detuning parameter. From Figs. 5a, 4b, we observe that the first and third mode amplitudes increase and become stable after the two saddle nodes as the forcing amplitude increases while a saturation phenomenon is not observed. The plots in Fig. 5c, 5d give more insight to what happens to the amplitude of the response while increasing the second force amplitude $F_{2 \Omega_{s}}$. In general, both amplitudes of the first and third modes increase with increasing the forcing value; however, the 
third mode shows an interesting behavior when the maximum amplitude passes through a sharp jump near $F_{2 \Omega_{s}}=6.5$ confirming the results in Fig. 4 where the hardening band dominates the response before the jump while a softening band due to the two-to-one internal resonance becomes dominant between $F_{2 \Omega_{s}}=6.5$ and $F_{2 \Omega_{s}}=8.5$. The additional force amplitude in this range does not contribute to an increase in the response amplitude of the third mode reaching a flat region, Fig. $5 \mathrm{~d}$, or saturation phenomenon in such range. Then, the amplitude of the hardening band becomes greater after increasing the forcing further. This indicates that the additional energy contribution of the second excitation force competes with that induced by the two-to-one internal resonance resulting in a suppression of the energy transfer due to internal resonance. We further investigate this phenomenon by plotting the loci of the Hopf bifurcation points as a function of the forcing amplitude $F_{2 \Omega_{s}}$ in Fig. 6.

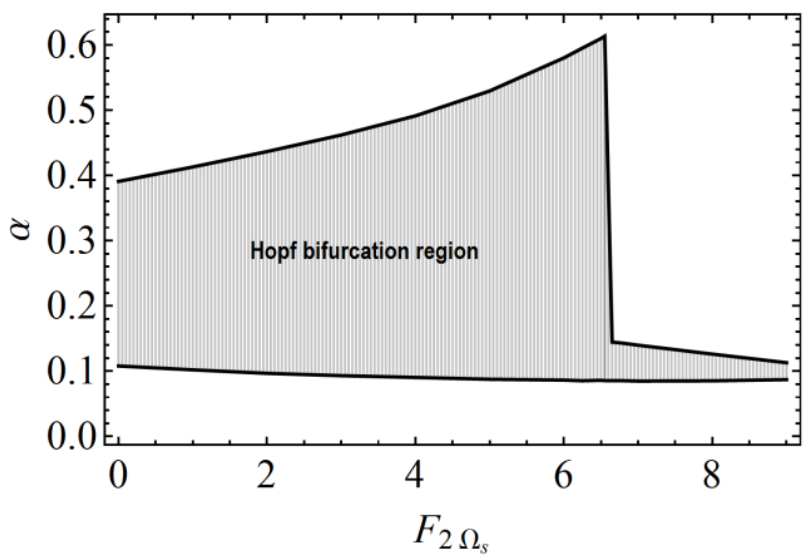

Figure 6: Loci of Hopf bifurcation for different values of forcing amplitude $F_{2 \Omega_{s}} . F_{\Omega_{s}}=8$, $\theta=62^{\circ}, \mu_{1}=0.08, \mu_{2}=0.08$.

The loci in Fig. 6 are obtained by examining the Jacobain of the system at different values of forcing amplitude. The result in Fig. 6 is an agreement with the trends observed in the force response curves showing that the Hopf bifurcation region increases as the forcing amplitude increases, which indicates that the 
energy from the external excitation increases the interaction due to the two-to-one internal resonance between forcing value of $0 \leq F_{2 \Omega_{s}} \leq 6.5$. Then, a sharp decrease (jump) is noted around $F_{2 \Omega_{s}}=6.5$ where the loci points are quenched to a smaller band at higher forcing amplitudes and are diminished at higher forcing amplitudes $F_{2 \Omega_{s}} \geq 9$. This phenomenon occurs due to competing effects of internal resonance activation and direct excitation of the third mode. Once the direct excitation forcing amplitude exceeded the threshold, it suppresses the nonlinear energy transfer via internal resonance where the jump is observed. It is noteworthy that for some cases, the response amplitude may possess more than the Hopf bifurcations shown in Fig. 6. Because the dynamical solution after the Hopf exhibits complex dynamical features, which may endanger the health of the riser, we use the Floquet theory with the shooting method [36] on the modulation equations to investigate the nature of the limit cycle and the solution in between the Hopf bifurcations. The results are depicted in Fig. 7.

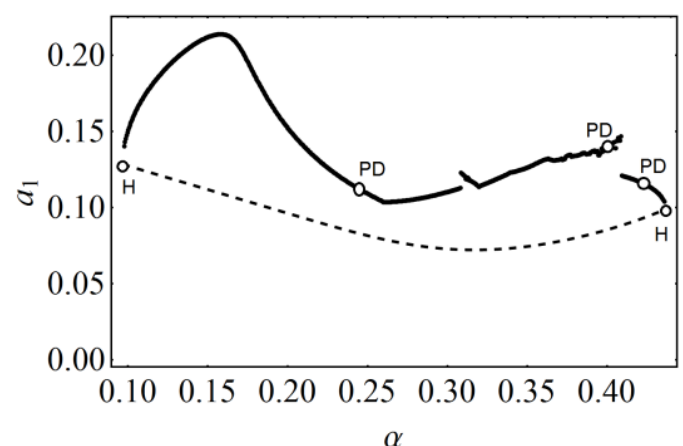

(a)

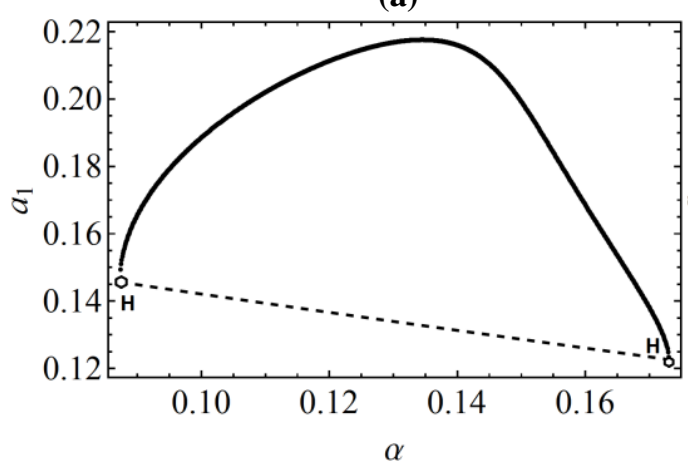

(c)

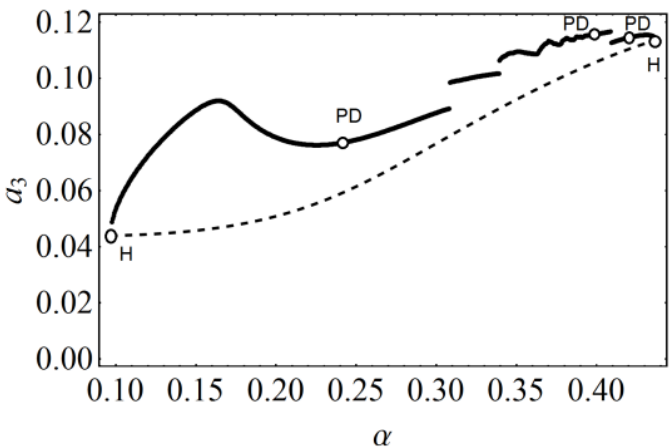

(b)

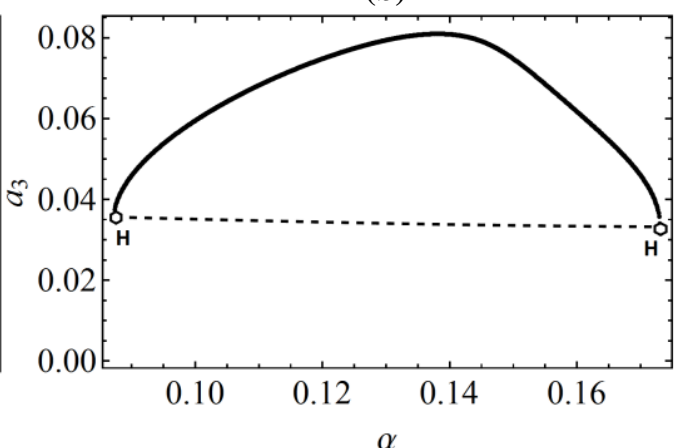

(d) 


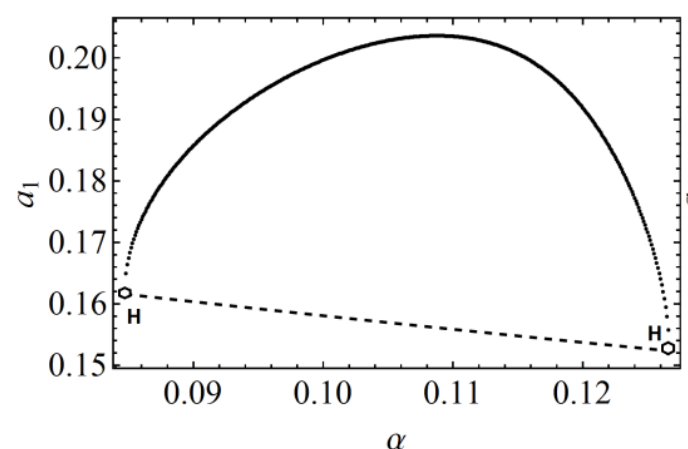

(e)

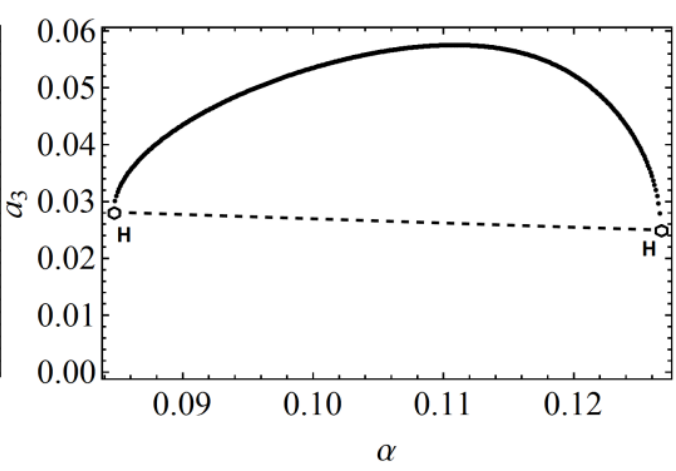

(f)

Figure 7: Frequency response curves of the limit cycle using the Floquet theory and the shooting method. $F_{\Omega_{s}}=8, \theta=62^{\circ}, \mu_{1}=0.08, \mu_{2}=0.08$. (- - -) Unstable solution obtained by the MTS method by solving the algebraic part of the modulation equations Eq. (15.1)-(15.4). (—) Solution by the Floquet theory using shooting method and sequential continuation on the modulation equations Eq. (15.1)-(15.4). (a,b) $F_{2 \Omega_{s}}=2$, (c,d) $F_{2 \Omega_{s}}=5$, (e,f) $F_{2 \Omega_{s}}=8$.

The results in Fig. 7 show the post-Hopf bifurcation response of the system at different values of excitation amplitude $F_{2 \Omega_{s}}$ represented by the limit cycle and the dynamical features where the Floquet multipliers are used to characterize their stability. Outside the Hopf bifurcation envelope, the response is characterized by a fixed point response similar to that observed in Fig. 3 and Fig. 4. Two limit cycles are born as a result of Hopf bifurcation. We consider here tracing and analyzing one of these solutions. First, in all the cases presented, we observe that the limit cycles are stable and, therefore, the Hopf bifurcations are supercritical. In addition, we observe at lower excitation amplitudes in Fig 7a, $7 \mathrm{~b}$ the limit cycle undergoes several period doubling bifurcations at $0.249,0.396$ and 0.429 with a Floquet multiplier exiting the unit circle through negative one. Also, the solution features several jumps and branches that can possibly be a route to chaotic motion. Further increasing the forcing amplitude reduces these complex dynamical features in Fig. $7 \mathrm{c}, 7 \mathrm{~d}$ where we observe that the solution has multiple Hopf bifurcations at $0.0872,0.173$ and also at $0.338,0.5296$. The response observed in these solutions has a stable limit cycle. A similar remark can be said on the limit cycle response in Fig. 7e, $7 \mathrm{f}$ obtained at higher excitation amplitude, i.e., post jump feature, 
where the obtained limit cycle is stable between the two Hopf bifurcation points 0.0847 and 0.1267 . Because such motion endangers the fatigue life of the riser, we further examine the dynamics in Fig. 8 and Fig. 9 by integrating the modulation equations to obtain the time history of the first and third mode amplitudes, their power spectra, phase space plots, and influence on the total deflection.
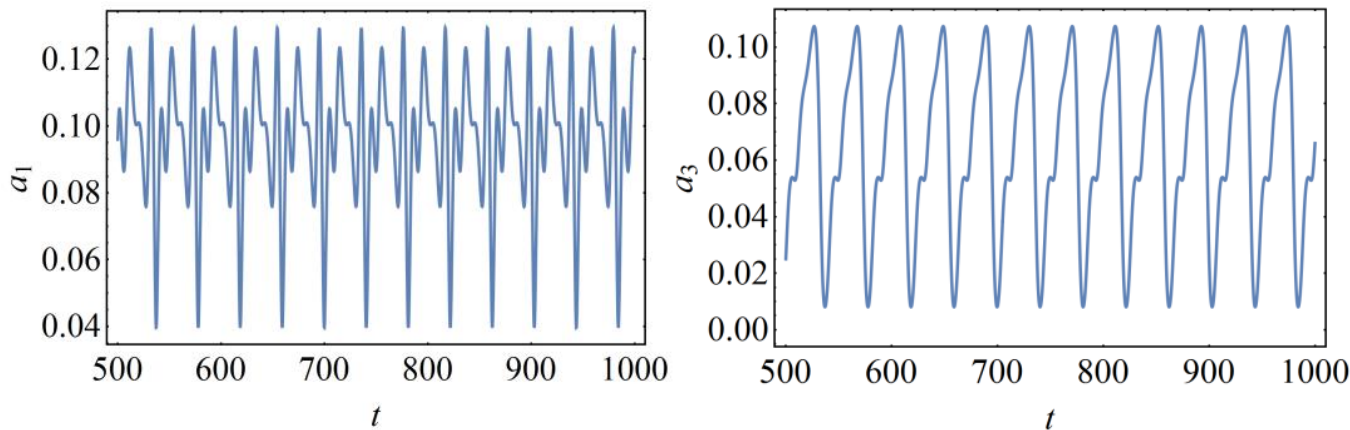

(a)

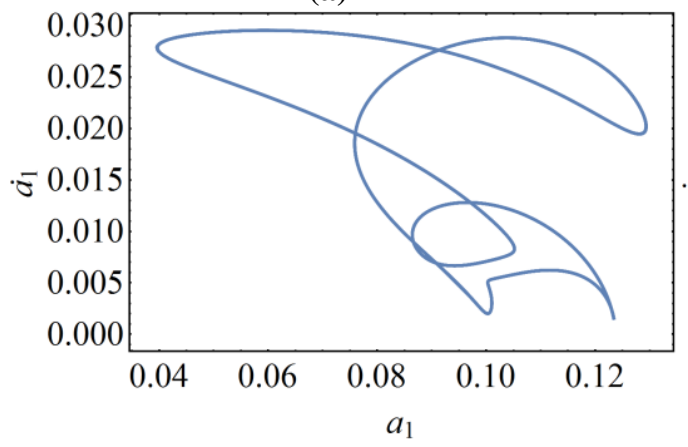

(b)

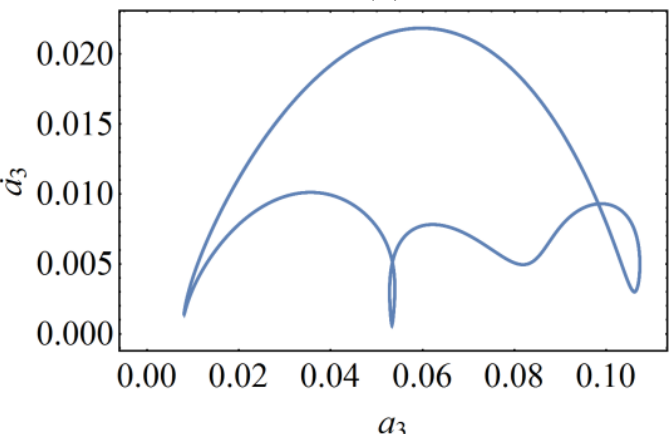

(c)

(d)

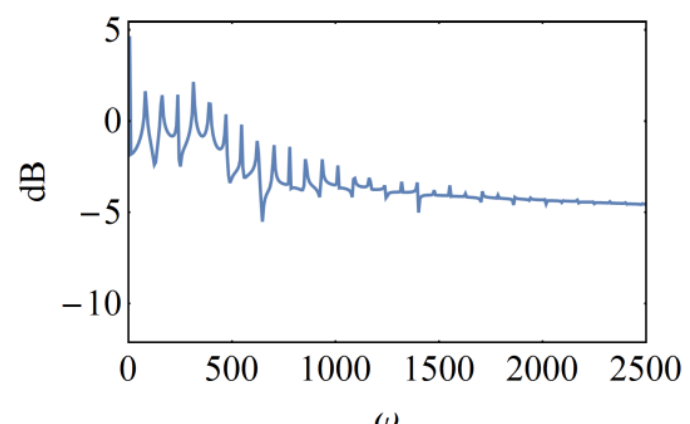

(e)

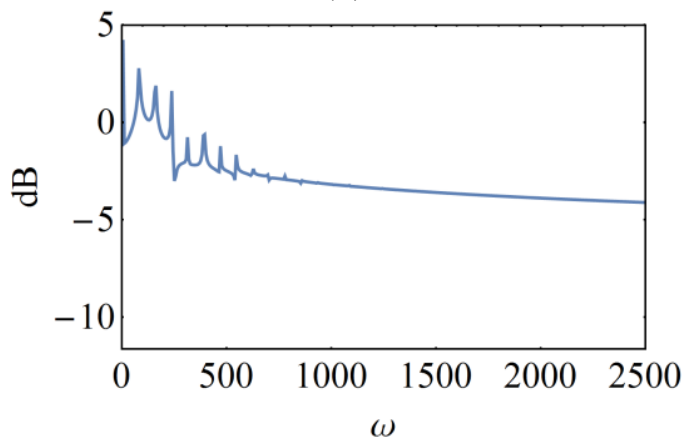

(f)

Figure 8: (a,b) Time histories of the first and third mode amplitudes. (c,d) Phase space plots. (e,f) Power spectra for $F_{\Omega_{s}}=8, \theta=62^{\circ}, \mu_{1}=0.08, \mu_{2}=0.08, F_{2 \Omega_{s}}=2, \alpha=0.342$. 


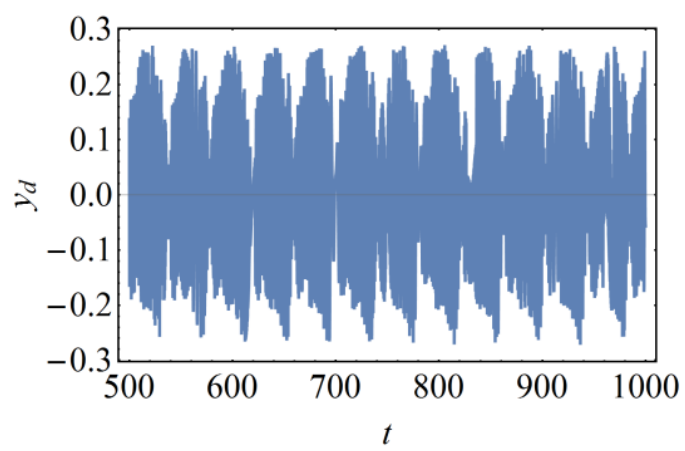

(a)

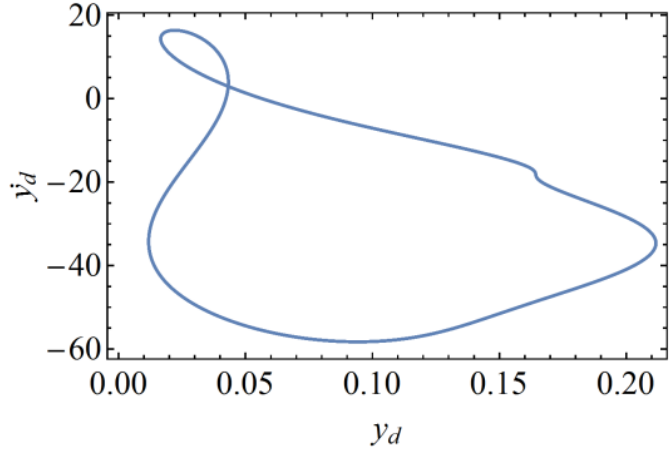

(b)

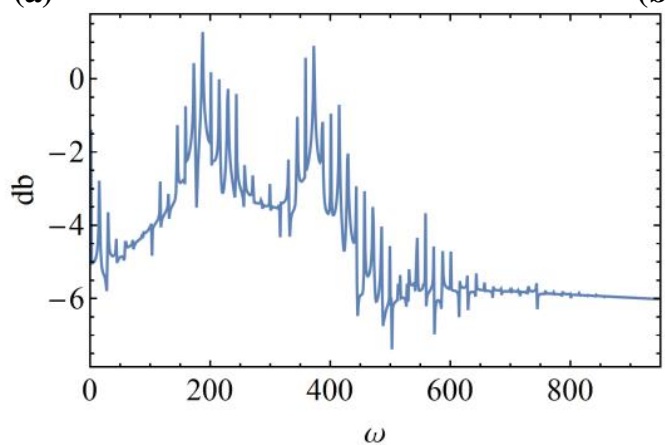

(c)

Figure 9: Time history (a) and the corresponding Poincare section (b) of the total deflection of the riser for $F_{\Omega_{s}}=8, \theta=62^{\circ}, \mu_{1}=0.08, \mu_{2}=0.08, F_{2 \Omega_{s}}=2, \alpha=0.342$.

The time histories in Fig. 8a, 8b corresponding to the amplitudes of the first and third modes demonstrate that multiple harmonics exist not only due to internal resonance, but also due to period doubling. This is confirmed by the presence of several loops in the phase space and the multiple peaks observed in the power spectrum. The amplitudes of the first and third modes are substituted in Eq. (16) to construct the full response of the riser shown in Fig. 9, which exhibits a quasiperiodic motion confirmed by the closed loop in the Poincare section in Fig. 9b. The quasiperiodicity is a result of the interaction of the periods of the response amplitude with the periods of the total response. This is due to the fact that the eigen frequencies of the limit cycle amplitudes $a_{1}$ and $a_{3}$ depend on the eigenvalues of the system of Eq. (15) which are demonstrated by the spectrum in Fig. 8. These eigenvalues become incommensurate when they are introduced in Eq. (16) because the solution $y_{d}$ has frequencies of $n-\Omega$. As an example, the major 
frequency values corresponding to the time history in Fig. 9a are 97.172, 186.452, $275.732,328.264,350.584,372.904,395.224$, and 417.544 which are incommensurable as demonstrated in Fig. 9c.

\subsection{Three-to-one internal resonance}

In the three-to-one internal resonance case, we consider the interaction between the first and the fifth mode. Therefore, the configuration angle is set to $\theta=15^{\circ}$ and the applied tension to $\bar{T}=1.2 \bar{\sigma}$ such that the ratio between the frequencies is three. Then, the result of the multiple time scales is compared to the solution obtained by solving Eq. (6) using five modes in the Galerkin procedure as shown in Fig. 10.

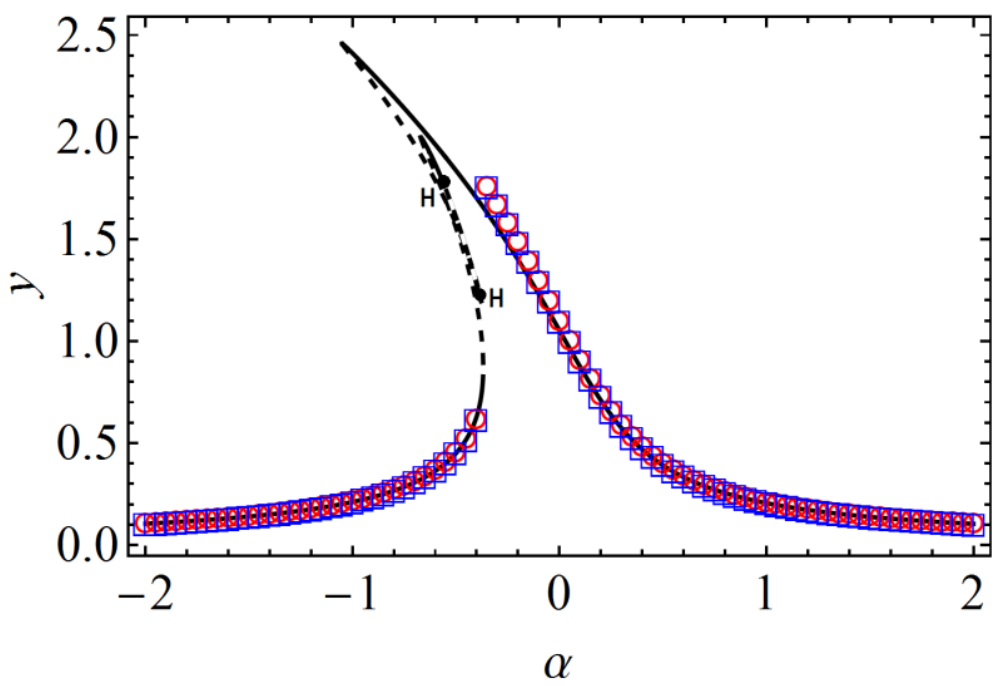

Figure 10: Frequency response curve of the riser deflection as varying the detuning parameter $\alpha$. $\theta=15^{\circ}, \quad \mu_{1}=0.08, \mu_{3}=0.08, F_{\Omega_{s}}=169, F_{3 \Omega_{s}}=50,(\square)$ Galerkin solution, forward sweep ( $\bigcirc$ ) Galerkin solution, backward sweep, (-) MTS stable solution, (- - - ) MTS unstable solution.

The frequency response curve in Fig. 10 shows good agreement between the multiple time scales and the Galerkin solution. The multiple time scales solution demonstrates the saddle node jump with interesting feature of the middle branch with the hysteresis, which causes computational difficulties in catching this branch using the Galerkin method. Further investigation is carried out by plotting 
the response amplitudes of the first and fifth modes for different excitation values of $F_{3 \Omega_{s}}$ in Fig. 11 and Fig. 12.

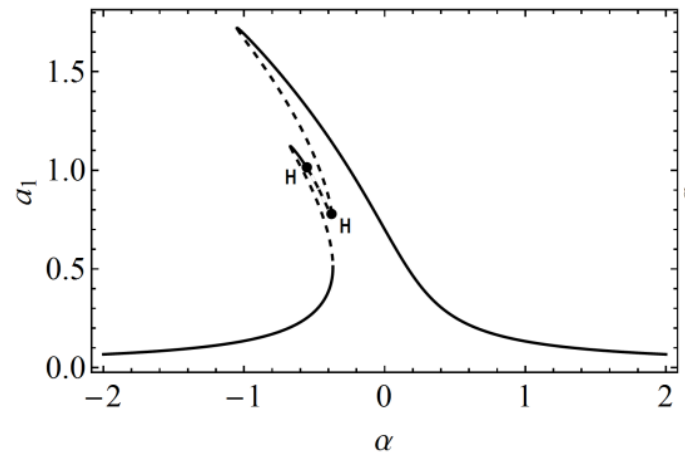

(a)

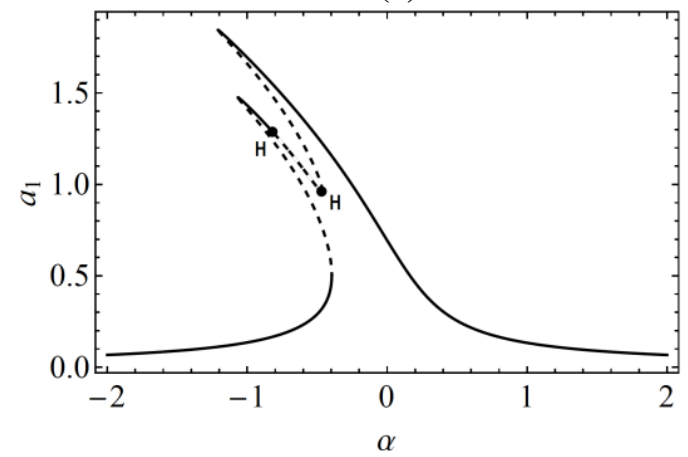

(c)

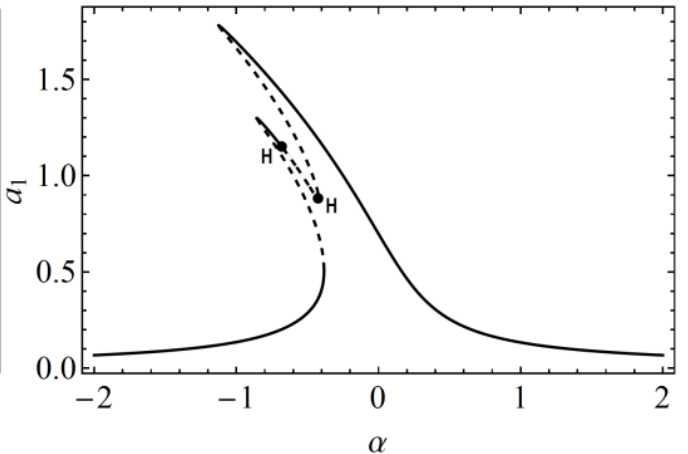

(b)

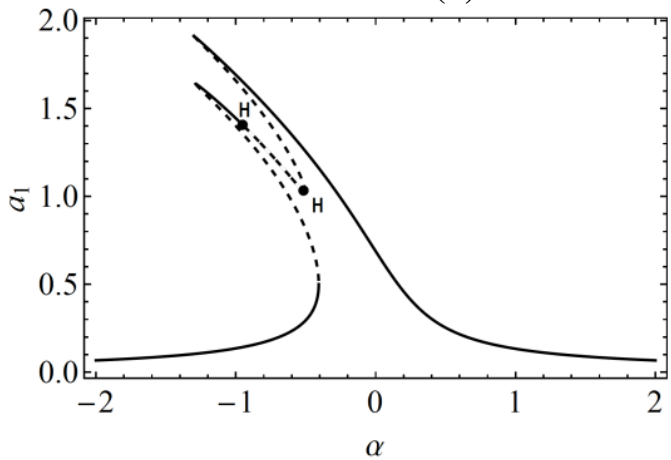

(d)

Figure 11: Frequency response curve of the first mode amplitude $a_{1}$ as varying the detuning parameter $\alpha . \theta=15^{\circ}, \mu_{1}=0.08, \mu_{2}=0.08, F_{\Omega_{s}}=169$. (a) $F_{3 \Omega_{s}}=50$, (b) $F_{3 \Omega_{s}}=100$, (c) $F_{3 \Omega_{s}}=150$, (d) $F_{3 \Omega_{s}}=200$.(•) H denotes Hopf bifurcation. (—) Stable solution, (- - ) unstable solution.

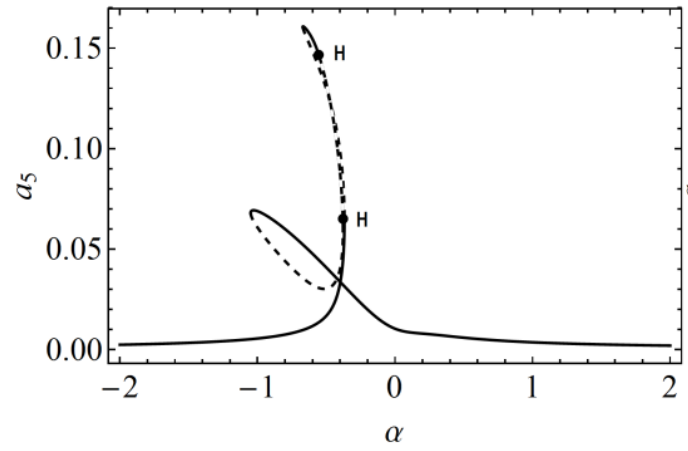

(a)

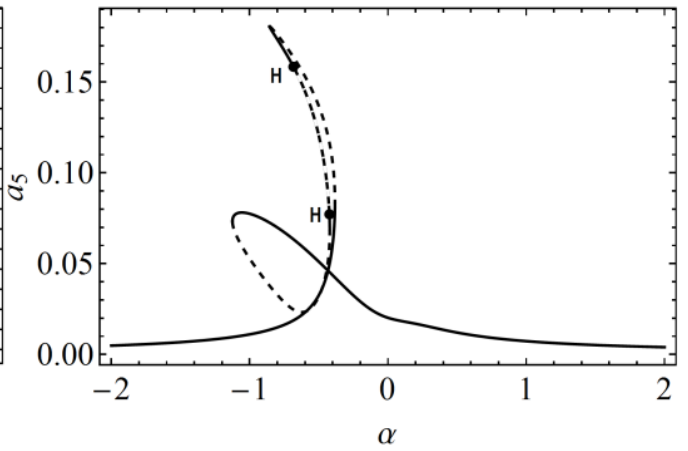

(b) 


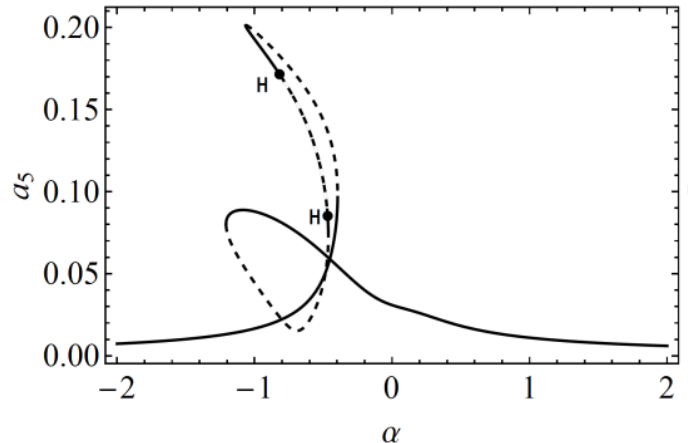

(c)

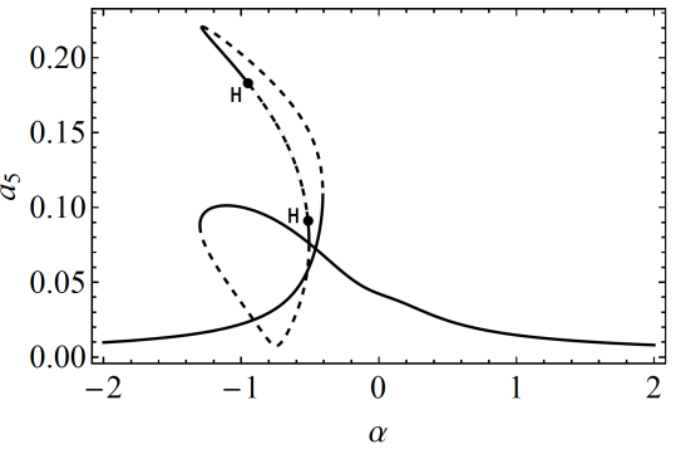

(d)

Figure 12: Frequency response curve of the fifth mode amplitude $a_{5}$ as varying the detuning parameter $\alpha . \theta=15^{\circ}, \mu_{1}=0.08, \mu_{2}=0.08, F_{\Omega_{s}}=169$. (a) $F_{3 \Omega_{s}}=50$, (b) $F_{3 \Omega_{s}}=100$, (c) $F_{3 \Omega_{s}}=150$, (d) $F_{3 \Omega_{s}}=200$. (• ) H denotes Hopf bifurcation. (—) Stable solution, (- - ) unstable solution.

The activation of the three-to-one internal resonance results into the emergence of a middle branch in the hysteresis zone of the amplitude of the first mode in Fig. 11. As the excitation amplitude $F_{3 \Omega_{s}}$ increases, two consequences are apparent in the results: First, the maximum amplitude of the response increases from Fig. 11a to $11 \mathrm{~b}$, and it is highest at Fig. 11d. Second, the bandwidth of the Hopf bifurcation zone increases. Due to the coupling by internal resonance, a similar behavior is observed in the response amplitude of the fifth mode in Fig. 12. The increase in the bandwidth of the Hopf zone is due to the competing softening effects arising from the first mode. This suggests that the second excitation has a role in increasing the internal resonance interaction between the modes. The frequency response curves corresponding to each case are plotted in Appendix C confirming the influence of the internal resonance interaction. A further investigation is carried out by plotting the loci of Hopf bifurcation bandwidth as varying the frequency detuning parameter in Fig. 13 


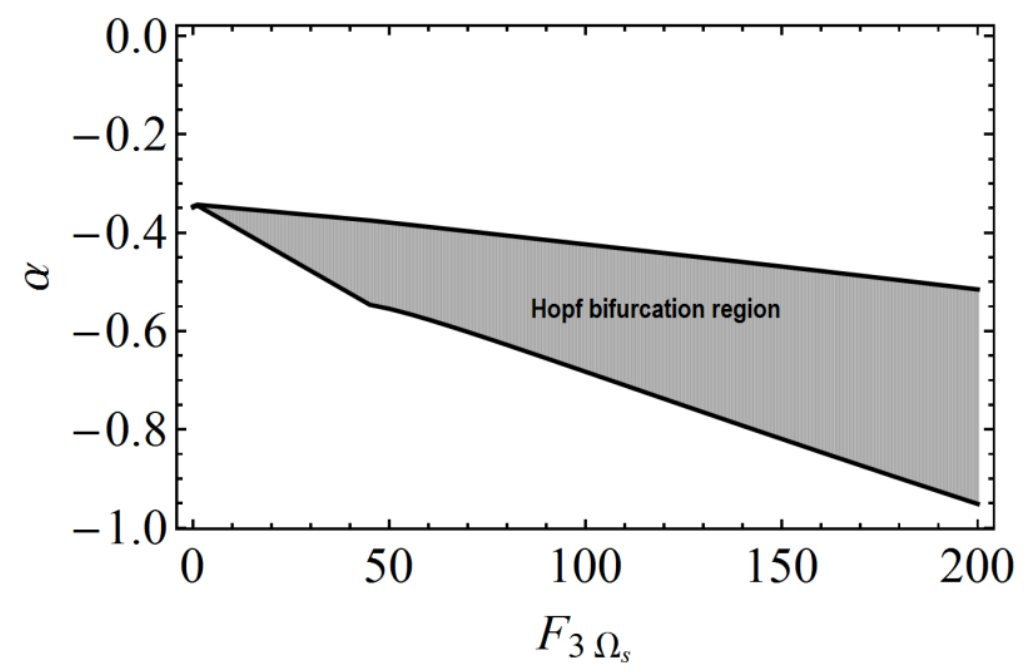

Figure 13: Loci of Hopf bifurcation at different values of forcing amplitude $F_{3 \Omega_{s}} . \theta=15^{\circ}$, $\mu_{1}=0.08, \mu_{2}=0.08, F_{\Omega_{s}}=169$.

In Fig. 13, we observe that increasing the excitation amplitude increases the bandwidth of the Hopf bifurcation band. In addition, a transition behavior is observed near $F_{3 \Omega_{s}}=40$ where the solution prior to that transition has interesting features between the stable and unstable branches similar to that observed in [28]. The further increase in the excitation results into diminishing these interesting features from the amplitude of the response. To illustrate what happens to the response amplitude at higher excitations, the frequency response curve of the response is plotted for $F_{3 \Omega_{s}}=282.69$ in Fig. 14 .
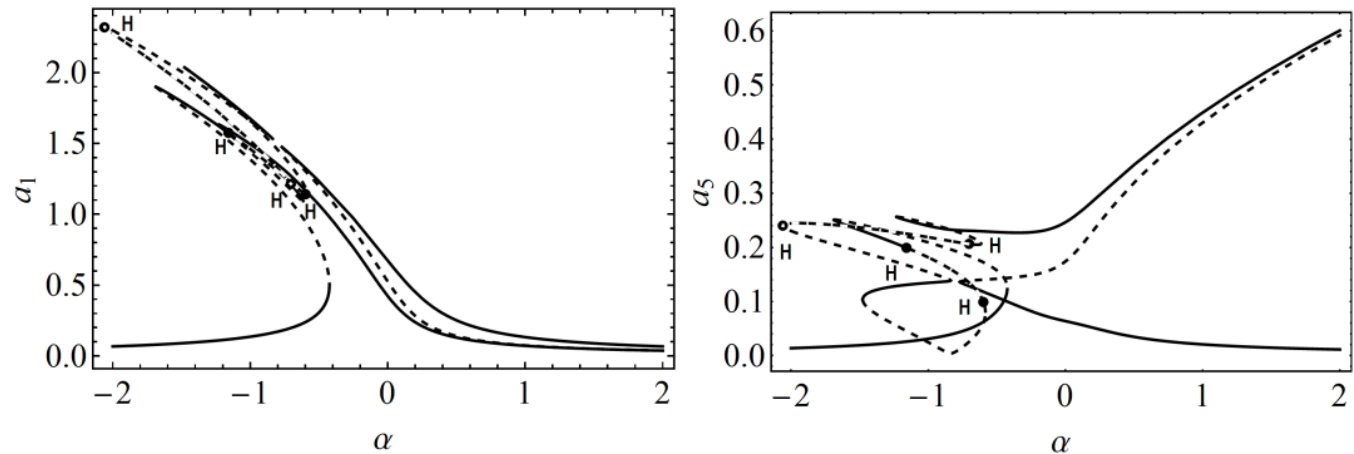

Figure 14: Frequency response curve of the first and fifth amplitudes as varying the detuning parameter $\alpha . \theta=15^{\circ}, \mu_{1}=0.08, \mu_{2}=0.08, F_{\Omega_{s}}=169, F_{3 \Omega_{s}}=282.69$. ( • ) H denotes Hopf bifurcation. (—) Stable solution, (- - ) unstable solution. 
Unlike the case of two-to-one internal resonance, in this case we observe from Fig. 14 that increasing the excitation amplitude further results into a more complicated response where multiple regions with Hopf bifurcation exist along with the intersection of solution of different branches. In addition, a transition behavior is observed where a new solution appears showing a hardening band in the amplitude of the fifth mode and softening in the first mode. This indicates that further increase in the excitation does not contribute only to internal resonance, but also directly forces the fifth mode. Because these features present a potential threat to the life of the riser, the dynamic behavior is further examined by integrating the modulation equations Eq. (20.1) to Eq. (20.4) and using the Floquet theory with the shooting method to obtain the response of the limit cycle and study its stability. The results are illustrated in Fig. 15.
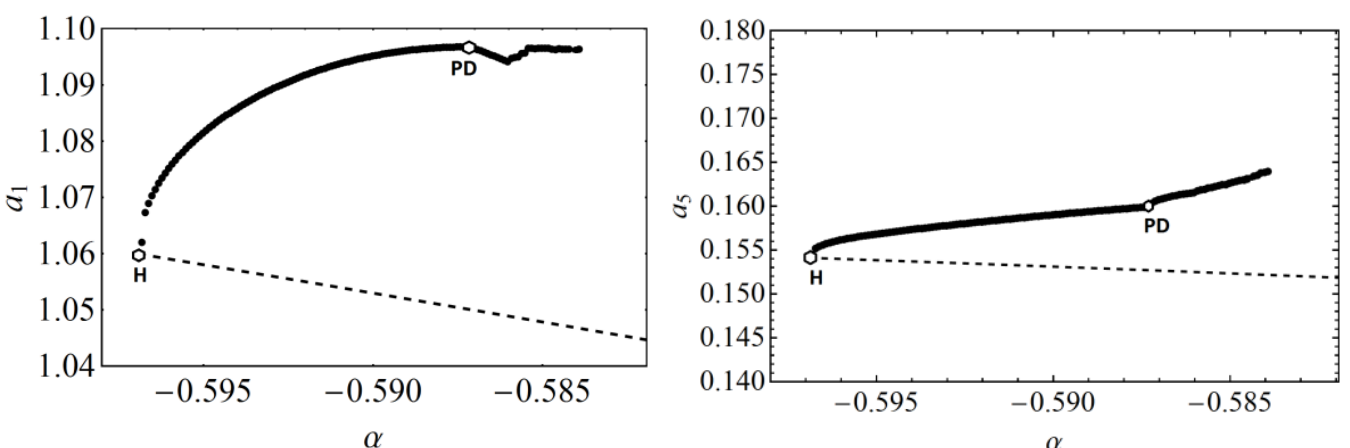

Figure 15: Frequency response curve of the limit cycle using the Floquet theory and the shooting method. $F_{\Omega_{s}}=169, F_{3 \Omega_{s}}=50, \theta=15^{\circ}, \mu_{1}=0.08, \mu_{2}=0.08$. (- - -) Unstable solution obtained by the MTS method by solving the algebraic part of the modulation equations Eq. (20.1)-(20.4). ( -) Solution by the Floquet theory using the shooting method and sequential continuation on the modulation equations Eq. (20.1)-(20.4).

In Fig. 15, we show a typical limit cycle observed in the vicinity of the Hopf bifurcation. Based on Floquet multipliers, the limit cycle is stable and, therefore, the Hopf bifurcation is supercritical. As increasing the frequency detuning parameter further, we notice the occurrence of period doubling bifurcation where one of the Floquet multipliers exits the unit circle through negative one. It is noteworthy that the solution is very sensitive to the initial conditions suggesting 
that the basin of attraction of the dynamic solution is very weak. The solution after period doubling shows complex dynamic features that affect the riser vibrations. The time histories, the phase space plots, and the power spectra corresponding to the first and fifth mode amplitudes are obtained by integrating the modulation equations and plotted in Fig. 16.

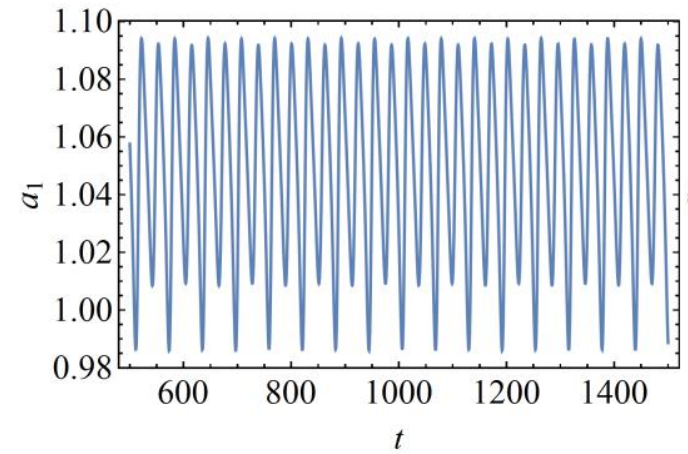

(a)

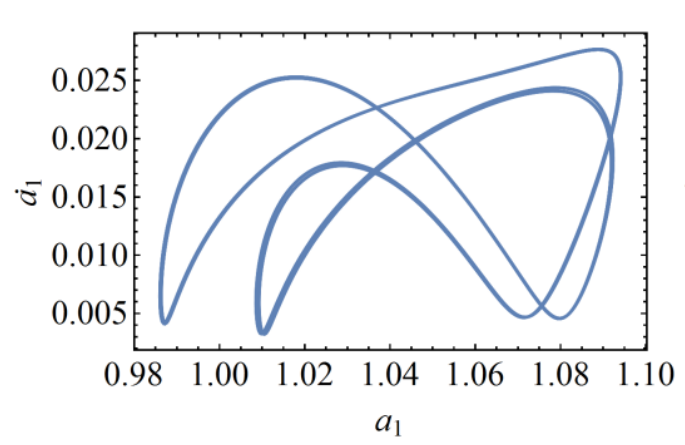

(c)

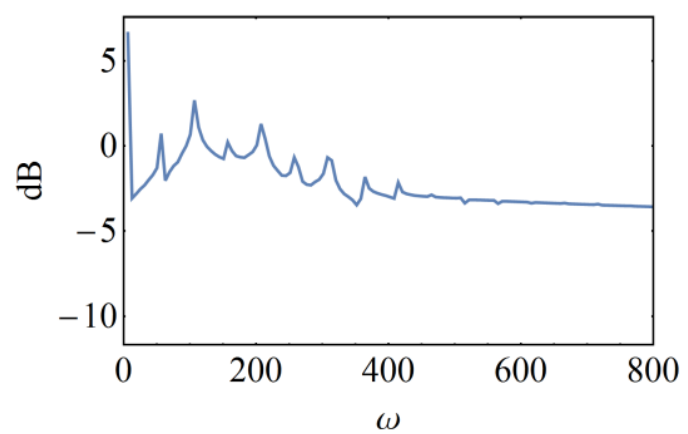

(e)

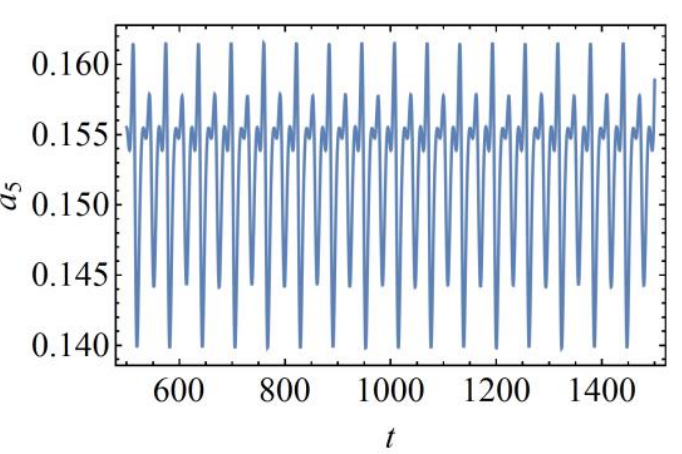

(b)

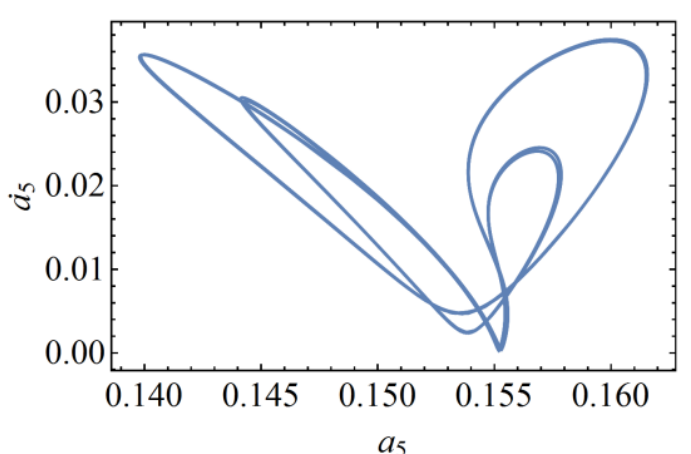

(d)

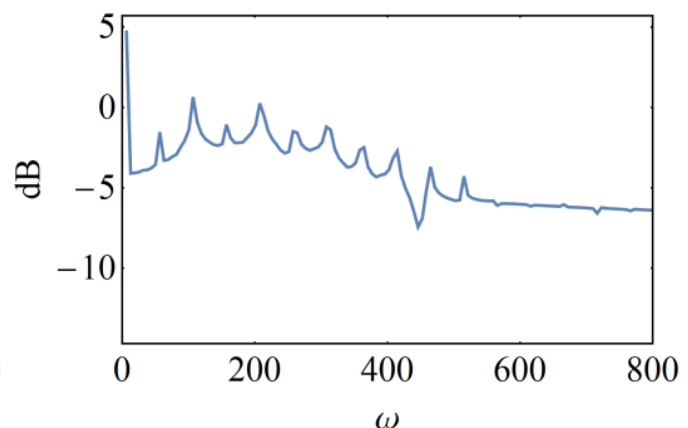

(f)

Figure 16: Time history and the corresponding Poincare sections of the first and fifth amplitudes for $F_{\Omega_{s}}=169, \theta=15^{\circ}, \mu_{1}=0.08, \mu_{2}=0.08, F_{3 \Omega_{s}}=50, \alpha=-0.585$.

The time histories in Fig. 16 show multiple harmonic periods in the response of the first and fifth modes amplitudes. The phase space plots demonstrate these 
different periods by the presence of the different orbits in the response of the first mode amplitude due to the period doubling and consequently in the response of the fifth mode amplitude due to the internal resonance interaction. Also, the power spectra demonstrate these multiple main peaks that are greater than zero indicating doubling of the period. To illustrate how these amplitudes affect the response of the riser, we substitute the responses of the first and fifth amplitude in Eq. (21) to obtain the total response of the riser, which is shown in Fig. 17.

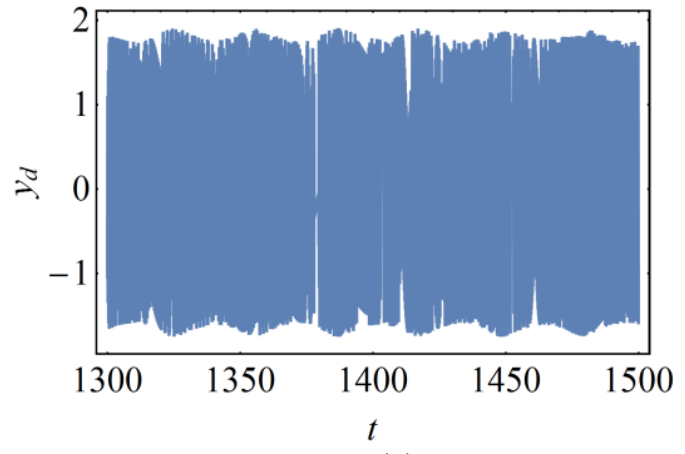

(a)

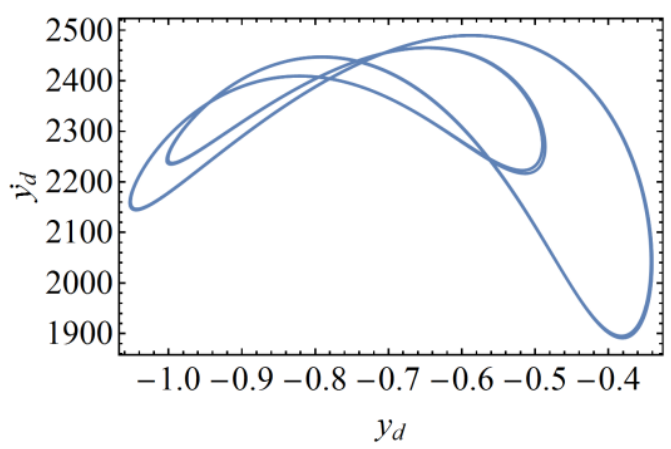

(b)

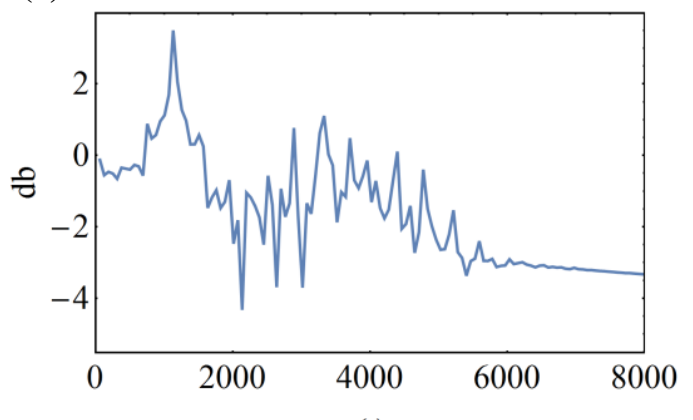

(c)

Figure 17: Time history (a) and the corresponding Poincare section (b) of the total deflection of the riser for $F_{\Omega_{s}}=169, \theta=15^{\circ}, \mu_{1}=0.08, \mu_{2}=0.08, F_{3 \Omega_{s}}=50, \alpha=-0.585$.

The interaction of the harmonics in the amplitude of the response due to period doubling with the main period of oscillation increases the complexity of the dynamics. We observe that the time history observed in Fig. 17 corresponds to a quasi-periodic motion. This is confirmed by the three closed loops in the Poincare section due to three-to-one internal resonance and the peaks in the power spectrum of Fig. 17c. The quasiperiodicity is a result of the interaction of the 
periods of the response amplitude with the periods of the total response similar to the case shown in the two-to-one internal resonance. Such motion in the riser structure can be catastrophic and detrimental from riser's fatigue point of view.

\section{Conclusions}

In conclusion, we investigated the influence of the multifrequency excitation on the response of the riser while considering two-to-one and three-toone internal resonances. The multiple time scales perturbation method is used to analyze the motion of the riser and showed good agreement when compared to the solution obtained by solving the original system using five modes in the Galerkin method. We observed that increasing the second excitation force in the two-to-one internal resonance case resulted into suppressing the energy transfer among the modes due to internal resonance and increasing the response via directly exciting each mode. On the other hand, the three-to-one internal resonance did not show signs of energy suppression. In addition, the dynamical response solution of the limit cycle after the Hopf bifurcation showed complex dynamical features such as period doubling with different jumps and branches. These dynamical features resulted into quasi-periodic motions of the system that threaten the riser life. A future prospective of this work is to analyze the riser motions considering internal resonances coupled with the vortex induced vibrations of the structure considering a Van der Pol oscillator model to capture the influence of the internal resonance and physical features of the vortex induced vibration phenomena. In addition, there is a need to explore the experimental aspects considering the static deformation with internal resonances to gain an in-depth insight of the nonlinear response of the riser. 


\section{Acknowledgements}

We acknowledge the financial support of King Abdullah Univeristy of Science and Technology and Saudi Aramco.

\section{Conflict of Interest}

The authors declare that they have no conflict of interest.

\section{Appendix A}

A.1 Definitions of the coefficients $R_{1}, R_{2}, K_{1}, K_{2}, K_{3}, K_{4}, \mu_{1}$, and $\mu_{2}$ for the case of Two-to-one internal resonance in Eq. (14.1) to Eq. (14.4)

$$
\begin{aligned}
& R_{1}=\int_{0}^{1} \phi_{m} \Lambda_{n m} \mathrm{~d} x \\
& \mu_{1}=\int_{0}^{1} c \phi_{m}^{2} \mathrm{~d} x \\
& \mu_{2}=\int_{0}^{1} c \phi_{n}^{2} \mathrm{~d} x \\
& R_{2}=\int_{0}^{1} \phi_{n}\left(\eta y_{s}^{\prime \prime}\left(\int_{0}^{1} \phi_{m}^{\prime 2} \mathrm{~d} x\right)+2 \eta \phi_{m}^{\prime \prime}\left(\int_{0}^{1} y_{s}^{\prime} \phi_{m}^{\prime} \mathrm{d} x\right)\right) \mathrm{d} x \\
& K_{1}=2 \eta \int_{0}^{1}\left(\int_{0}^{1} \phi_{n}^{\prime} \psi_{1}^{\prime} \mathrm{d} x\right) \phi_{m} y_{s}^{\prime \prime} \mathrm{d} x+2 \eta \int_{0}^{1}\left(\int_{0}^{1} \phi_{m}^{\prime} \psi_{4}^{\prime} \mathrm{d} x\right) \phi_{m} y_{s}^{\prime \prime} \mathrm{d} x+2 \eta \int_{0}^{1}\left(\int_{0}^{1}\left(\phi_{n}^{\prime}\right)^{2} \mathrm{~d} x\right) \phi_{m} \phi_{m}{ }^{\prime \prime} \mathrm{d} x \\
& +2 \eta \int_{0}^{1}\left(\int_{0}^{1} y_{s}^{\prime} \psi_{4}^{\prime} \mathrm{d} x\right) \phi_{m} \phi_{m}^{\prime \prime} \mathrm{d} x+4 \eta \int_{0}^{1}\left(\int_{0}^{1} \phi_{m}^{\prime} \phi_{n}^{\prime} \mathrm{d} x\right) \phi_{m} \phi_{n}^{\prime \prime} \mathrm{d} x+2 \eta \int_{0}^{1}\left(\int_{0}^{1} y_{s}^{\prime} \psi_{1}^{\prime} \mathrm{d} x\right) \phi_{m} \phi_{n}^{\prime \prime} \mathrm{d} x \\
& +2 \eta \int_{0}^{1}\left(\int_{0}^{1} y_{s}^{\prime} \phi_{n}^{\prime} \mathrm{d} x\right) \phi_{m} \psi_{1}^{\prime \prime} \mathrm{d} x+2 \eta \int_{0}^{1}\left(\int_{0}^{1} y_{s}^{\prime} \phi_{m}^{\prime} \mathrm{d} x\right) \phi_{m} \psi_{4}^{\prime \prime} \mathrm{d} x \\
& K_{2}=2 \eta \int_{0}^{1}\left(\int_{0}^{1} \phi_{m}^{\prime} \psi_{3}^{\prime} \mathrm{d} x\right) \phi_{m} y_{s}^{\prime \prime} \mathrm{d} x+3 \eta \int_{0}^{1}\left(\int_{0}^{1}\left(\phi_{m}^{\prime}\right)^{2} \mathrm{~d} x\right) \phi_{m} \phi_{m}^{\prime \prime} \mathrm{d} x+2 \eta \int_{0}^{1}\left(\int_{0}^{1} y_{s}^{\prime} \psi_{3}^{\prime} \mathrm{d} x\right) \phi_{m} \phi_{m}{ }^{\prime \prime} \mathrm{d} x \\
& +2 \eta \int_{0}^{1}\left(\int_{0}^{1} y_{s}^{\prime} \phi_{m}^{\prime} \mathrm{d} x\right) \phi_{m} \psi_{3}^{\prime \prime} \mathrm{d} x
\end{aligned}
$$




$$
\begin{aligned}
K_{3} & =2 \eta \int_{0}^{1}\left(\int_{0}^{1} \phi_{m}^{\prime} \psi_{1}^{\prime} \mathrm{d} x\right) \phi_{n} y_{s}^{\prime \prime} \mathrm{d} x+2 \eta \int_{0}^{1}\left(\int_{0}^{1} \phi_{n}^{\prime} \psi_{3}^{\prime} \mathrm{d} x\right) \phi_{n} y_{s}^{\prime \prime} \mathrm{d} x+4 \eta \int_{0}^{1}\left(\int_{0}^{1} \phi_{m}^{\prime} \phi_{n}^{\prime} \mathrm{d} x\right) \phi_{n} \phi_{m}^{\prime \prime} \mathrm{d} x \\
& +2 \eta \int_{0}^{1}\left(\int_{0}^{1} y_{s}^{\prime} \psi_{1}^{\prime} \mathrm{d} x\right) \phi_{n} \phi_{m}^{\prime \prime} \mathrm{d} x+2 \eta \int_{0}^{1}\left(\int_{0}^{1}\left(\phi_{m}^{\prime}\right)^{2} \mathrm{~d} x\right) \phi_{n} \phi_{n}^{\prime \prime} \mathrm{d} x+2 \eta \int_{0}^{1}\left(\int_{0}^{1} y_{s}^{\prime} \psi_{3}^{\prime} \mathrm{d} x\right) \phi_{n} \phi_{n}^{\prime \prime} \mathrm{d} x \\
& +2 \eta \int_{0}^{1}\left(\int_{0}^{1} y_{s}^{\prime} \phi_{m}^{\prime} \mathrm{d} x\right) \phi_{n} \psi_{1}^{\prime \prime} \mathrm{d} x+2 \eta \int_{0}^{1}\left(\int_{0}^{1} y_{s}^{\prime} \phi_{n}^{\prime} \mathrm{d} x\right) \phi_{n} \psi_{3}^{\prime \prime} \mathrm{d} x \\
K_{4} & =2 \eta \int_{0}^{1}\left(\int_{0}^{1} \phi_{n}^{\prime} \psi_{2}^{\prime} \mathrm{d} x\right) \phi_{n} y_{s}^{\prime \prime} \mathrm{d} x+2 \eta \int_{0}^{1}\left(\int_{0}^{1} \phi_{n}^{\prime} \psi_{4}^{\prime} \mathrm{d} x\right) \phi_{n} y_{s}^{\prime \prime} \mathrm{d} x+3 \eta \int_{0}^{1}\left(\int_{0}^{1}\left(\phi_{n}^{\prime}\right)^{2} \mathrm{~d} x\right) \phi_{n} \phi_{n}^{\prime \prime} \mathrm{d} x \\
& +2 \eta \int_{0}^{1}\left(\int_{0}^{1} y_{s}^{\prime} \psi_{2}^{\prime} \mathrm{d} x\right) \phi_{n} \phi_{n}^{\prime \prime} \mathrm{d} x+2 \eta \int_{0}^{1}\left(\int_{0}^{1} y_{s}^{\prime} \psi_{4}^{\prime} \mathrm{d} x\right) \phi_{n} \phi_{n}^{\prime \prime} \mathrm{d} x+2 \eta \int_{0}^{1}\left(\int_{0}^{1} y_{s}^{\prime} \phi_{n}^{\prime} \mathrm{d} x\right) \phi_{n} \psi_{2}^{\prime \prime} \mathrm{d} x \\
& +2 \eta \int_{0}^{1}\left(\int_{0}^{1} y_{s}^{\prime} \phi_{n}^{\prime} \mathrm{d} x\right) \phi_{n} \psi_{4}^{\prime \prime} \mathrm{d} x
\end{aligned}
$$

A.2 Definitions of the coefficients $R_{1}, R_{2}, R_{3}, K_{1}, K_{2}, K_{3}, \mu_{1}$, and $\mu_{2}$ for the case of Three-to-one internal resonance in Eq. (19.1) to Eq. (19.4)

$R_{1}=\frac{1}{8} \int_{0}^{1} \phi_{m} \psi_{1} d x, \quad R_{2}=\frac{1}{8} \int_{0}^{1} \phi_{m} \psi_{2} d x, \quad R_{3}=\frac{1}{8} \int_{0}^{1} \phi_{m} \psi_{5} d x$

$K_{1}=\frac{1}{8} \int_{0}^{1} \phi_{n} \psi_{3} d x, \quad K_{2}=\frac{1}{8} \int_{0}^{1} \phi_{n} \psi_{4} d x, \quad K_{3}=\frac{1}{8} \int_{0}^{1} \phi_{n} \psi_{6} d x$

$$
\mu_{1}=\frac{1}{2} \int_{0}^{1} c \phi_{m}{ }^{2} d x, \quad \mu_{2}=\frac{1}{2} \int_{0}^{1} c \phi_{n}^{2} d x
$$

where $\psi_{i}$ are functions defined by

$$
\begin{aligned}
\psi_{1}(x) & =2 \eta\left(\int_{0}^{1} \Pi_{1 m}{ }^{\prime} \phi_{m}{ }^{\prime} \mathrm{d} x\right) y_{s}{ }^{\prime \prime}+2 \eta\left(\int_{0}^{1} \Pi_{2 m}{ }^{\prime} \phi_{m}{ }^{\prime} \mathrm{d} x\right) y_{s}{ }^{\prime \prime}+2 \eta\left(\int_{0}^{1} y_{s}{ }^{\prime} \phi_{m}{ }^{\prime} \mathrm{d} x\right) \Pi_{1 m}{ }^{\prime \prime} \\
& +2 \eta\left(\int_{0}^{1} y_{s}^{\prime} \phi_{m}^{\prime} \mathrm{d} x\right) \Pi_{2 m}{ }^{\prime \prime}+2 \eta\left(\int_{0}^{1} y_{s}^{\prime} \Pi_{1 m}{ }^{\prime} \mathrm{d} x\right) \phi_{m}{ }^{\prime \prime}+2 \eta\left(\int_{0}^{1} y_{s}{ }_{s}^{\prime} \Pi_{2 m}{ }^{\prime} \mathrm{d} x\right) \phi_{m}{ }^{\prime \prime} \\
& +3 \eta\left(\int_{0}^{1}\left(\phi_{m}{ }^{\prime}\right)^{2} \mathrm{~d} x\right) \phi_{m}{ }^{\prime \prime}
\end{aligned}
$$




$$
\begin{aligned}
& \psi_{2}(x)=2 \eta\left(\int_{0}^{1} \Pi_{2 n}{ }^{\prime} \phi_{m}^{\prime} \mathrm{d} x\right) y_{s}^{\prime \prime}+2 \eta\left(\int_{0}^{1} \Pi_{1 m n}{ }^{\prime} \phi_{n}^{\prime} \mathrm{d} x\right) y_{s}^{\prime \prime}+2 \eta\left(\int_{0}^{1} \Pi_{2 m n}{ }^{\prime} \phi_{n}^{\prime} \mathrm{d} x\right) y_{s}^{\prime \prime} \\
& +2 \eta\left(\int_{0}^{1} y_{s}^{\prime} \phi_{m}^{\prime} \mathrm{d} x\right) \Pi_{2 n}^{\prime \prime}+2 \eta\left(\int_{0}^{1} y_{s}^{\prime} \phi_{n}^{\prime} \mathrm{d} x\right) \Pi_{1 m n}{ }^{\prime \prime}+2 \eta\left(\int_{0}^{1} y_{s}^{\prime} \phi_{n}^{\prime} x\right) \Pi_{2 m n}{ }^{\prime \prime} \\
& +2 \eta\left(\int_{0}^{1} y_{s}^{\prime} \Pi_{2 n}^{\prime} \mathrm{d} x\right) \phi_{m}^{\prime \prime}+2 \eta\left(\int_{0}^{1}\left(\phi_{n}^{\prime}\right)^{2} \mathrm{~d} x\right) \phi_{m}^{\prime \prime}+2 \eta\left(\int_{0}^{1} y_{s}^{\prime} \Pi_{1 m n}{ }^{\prime} \mathrm{d} x\right) \phi_{n}^{\prime \prime} \\
& +2 \eta\left(\int_{0}^{1} y_{s}^{\prime} \Pi_{2 m n}^{\prime} \mathrm{d} x\right) \phi_{n}^{\prime \prime}+4 \eta\left(\int_{0}^{1} \phi_{m}^{\prime} \phi_{n}^{\prime} \mathrm{d} x\right) \phi_{n}^{\prime \prime} \\
& \psi_{3}(x)=2 \eta\left(\int_{0}^{1} \Pi_{1 n}{ }^{\prime} \phi_{n}^{\prime} \mathrm{d} x\right) y_{s}^{\prime \prime}+2 \eta\left(\int_{0}^{1} \Pi_{2 n}^{\prime} \phi_{n}^{\prime} \mathrm{d} x\right) y_{s}^{\prime \prime}+2 \eta\left(\int_{0}^{1} y_{s}^{\prime} \phi_{n}^{\prime} \mathrm{d} x\right) \Pi_{1 n}{ }^{\prime \prime} \\
& +2 \eta\left(\int_{0}^{1} y_{s}^{\prime} \phi_{n}^{\prime} \mathrm{d} x\right) \Pi_{2 n}{ }^{\prime \prime}+2 \eta\left(\int_{0}^{1} y_{s}^{\prime} \Pi_{1 n}^{\prime} \mathrm{d} x\right) \phi_{n}^{\prime \prime}+2 \eta\left(\int_{0}^{1} y_{s}^{\prime} \Pi_{2 n}^{\prime} \mathrm{d} x\right) \phi_{n}^{\prime \prime} \\
& +3 \eta\left(\int_{0}^{1}\left(\phi_{n}^{\prime}\right)^{2} \mathrm{~d} x\right) \phi_{n}^{\prime \prime} \\
& \psi_{4}(x)=2 \eta\left(\int_{0}^{1} \Pi_{1 m n}{ }^{\prime} \phi_{m}{ }^{\prime} \mathrm{d} x\right) y_{s}^{\prime \prime}+2 \eta\left(\int_{0}^{1} \Pi_{2 m n}{ }^{\prime} \phi_{m}{ }^{\prime} \mathrm{d} x\right) y_{s}^{\prime \prime}+2 \eta\left(\int_{0}^{1} \Pi_{2 m}{ }^{\prime} \phi_{n}^{\prime} \mathrm{d} x\right) y_{s}^{\prime \prime} \\
& +4 \eta\left(\int_{0}^{1} \phi_{m}^{\prime} \phi_{n}^{\prime} \mathrm{d} x\right) \phi_{m}^{\prime \prime}+2 \eta\left(\int_{0}^{1} y_{s}^{\prime} \Pi_{1 m n}{ }^{\prime} \mathrm{d} x\right) \phi_{m}^{\prime \prime}+2 \eta\left(\int_{0}^{1} y_{s}^{\prime} \Pi_{2 m n}{ }^{\prime} \mathrm{d} x\right) \phi_{m}{ }^{\prime \prime} \\
& +2 \eta\left(\int_{0}^{1} y_{s}^{\prime} \Pi_{2 m}^{\prime} \mathrm{d} x\right) \phi_{n}^{\prime \prime}+2 \eta\left(\int_{0}^{1}\left(\phi_{m}^{\prime}\right)^{2} \mathrm{~d} x\right) \phi_{n}^{\prime \prime}+2 \eta\left(\int_{0}^{1} y_{s}^{\prime} \phi_{n}^{\prime} \mathrm{d} x\right) \Pi_{2 m}^{\prime \prime} \\
& +2 \eta\left(\int_{0}^{1} y_{s}^{\prime} \phi_{m}^{\prime} \mathrm{d} x\right) \Pi_{1 m n}{ }^{\prime \prime}+2 \eta\left(\int_{0}^{1} y_{s}^{\prime} \phi_{m}^{\prime} \mathrm{d} x\right) \Pi_{2 m n}{ }^{\prime \prime} \\
& \psi_{5}(x)=2 \eta y_{s}^{\prime \prime}\left(\int_{0}^{1} \phi_{m}^{\prime} \Pi_{1 m n}{ }^{\prime} \mathrm{d} x\right)+2 \eta y_{s}^{\prime \prime}\left(\int_{0}^{1} \Pi_{1 m}^{\prime} \phi_{n}^{\prime} \mathrm{d} x\right)+2 \eta \Pi_{1 m}{ }^{\prime \prime}\left(\int_{0}^{1} \phi_{n}^{\prime} y_{s}^{\prime} \mathrm{d} x\right) \\
& +2 \eta \Pi_{1 m n}{ }^{\prime \prime}\left(\int_{0}^{1} \phi_{m}^{\prime} y_{s}^{\prime} \mathrm{d} x\right)+2 \eta \phi_{m}^{\prime \prime}\left(\int_{0}^{1} y_{s}^{\prime} \Pi_{1 m n}^{\prime} \mathrm{d} x\right)+2 \eta \phi_{n}^{\prime \prime}\left(\int_{0}^{1} \Pi_{1 m}^{\prime} y_{s}^{\prime} \mathrm{d} x\right) \\
& +2 \eta \phi_{m}^{\prime \prime}\left(\int_{0}^{1} \phi_{m}^{\prime} \phi_{n}^{\prime} \mathrm{d} x\right)+\eta \phi_{n}^{\prime \prime}\left(\int_{0}^{1}\left(\phi_{m}^{\prime}\right)^{2} \mathrm{~d} x\right) \\
& \psi_{6}(x)=2 \eta y_{s}^{\prime \prime}\left(\int_{0}^{1} \Pi_{1 m}{ }^{\prime} \phi_{m}^{\prime} \mathrm{d} x\right)+2 \eta \Pi_{1 m}{ }^{\prime \prime}\left(\int_{0}^{1} \phi_{m}^{\prime} y_{s}^{\prime} \mathrm{d} x\right)+2 \eta \phi_{m}^{\prime \prime}\left(\int_{0}^{1} \Pi_{1 m}{ }^{\prime} y_{s}^{\prime} \mathrm{d} x\right) \\
& +\eta \phi_{m}^{\prime \prime}\left(\int_{0}^{1}\left(\phi_{m}^{\prime}\right)^{2} \mathrm{~d} x\right)
\end{aligned}
$$

33 


\section{Appendix B}

B.1 Cartesian form of the modulation equations pertaining to two-to-one internal resonance.

$$
\begin{aligned}
& \dot{p}_{m}=-\frac{\mu_{1}}{2} p_{m}+\left(\frac{R_{1} F_{2 \Omega_{s}}}{8 \omega_{m} \omega_{n}^{2}}-\alpha-\frac{\mu_{1}^{2}}{8 \omega_{m}}\right) q_{m}-\frac{R_{1} F_{\Omega_{s}}}{8 \omega_{m}^{3}} q_{n}+\frac{\mu_{1} F_{\Omega_{s}}}{4 \omega_{m}^{2}} \\
& +\left(\frac{\mu_{1} R_{1}}{8 \omega_{m}^{2}}-\frac{\mu_{1} R_{1}}{16 \omega_{m} \omega_{n}}\right)\left(p_{m} p_{n}+q_{m} q_{n}\right)+\frac{R_{1}}{4 \omega_{m}}\left(q_{m} p_{n}-p_{m} q_{n}\right) \\
& -\left(\frac{K_{1}}{8 \omega_{m}}+\frac{R_{1}^{2}}{32 \omega_{m}^{3}}\right)\left(q_{m} p_{n}^{2}+q_{m} q_{n}^{2}\right)-\left(\frac{K_{2}}{8 \omega_{m}}+\frac{R_{1} R_{2}}{32 \omega_{m} \omega_{n}^{2}}\right)\left(p_{m}^{2} q_{m}+q_{m}^{3}\right) \\
& \dot{q}_{m}=-\frac{\mu_{1}}{2} q_{m}+\left(\alpha+\frac{R_{1} F_{2 \Omega_{s}}}{8 \omega_{m} \omega_{n}^{2}}+\frac{\mu_{1}^{2}}{8 \omega_{m}}\right) p_{m}+\frac{R_{1} F_{\Omega_{s}}}{8 \omega_{m}^{3}} p_{n}+\frac{F_{\Omega_{s}}}{\omega_{m}} \\
& +\frac{R_{1}}{4 \omega_{m}}\left(p_{m} p_{n}+q_{m} q_{n}\right)+\left(\frac{\mu_{1} R_{1}}{8 \omega_{m}^{2}}-\frac{\mu_{1} R_{1}}{16 \omega_{m} \omega_{n}}\right)\left(p_{m} q_{n}-q_{m} p_{n}\right) \\
& +\left(\frac{K_{1}}{8 \omega_{m}}+\frac{R_{1}^{2}}{32 \omega_{m}^{3}}\right)\left(p_{m} q_{n}^{2}+p_{m} p_{n}^{2}\right)+\left(\frac{K_{2}}{8 \omega_{m}}+\frac{R_{1} R_{2}}{32 \omega_{m} \omega_{n}^{2}}\right)\left(p_{m} q_{m}^{2}+p_{m}^{3}\right) \\
& \dot{p}_{n}=-\frac{\mu_{1}}{2} p_{n}+\left(\delta-2 \alpha-\frac{\mu_{1}^{2}}{8 \omega_{n}}\right) q_{n}-\frac{R_{2} F_{\Omega_{s}}}{4 \omega_{m}^{2} \omega_{n}} q_{m}+\frac{\mu_{1} F_{2 \Omega_{s}}}{4 \omega_{n}^{2}} \\
& -\frac{R_{2}}{2 \omega_{n}} p_{m} q_{m}-\left(\frac{K_{3}}{8 \omega_{n}}+\frac{R_{1} R_{2}}{16 \omega_{m}^{2} \omega_{n}}\right)\left(p_{m}^{2} q_{n}+q_{m}^{2} q_{n}\right)-\frac{K_{4}}{8 \omega_{n}}\left(p_{n}^{2} q_{n}+q_{n}^{3}\right) \\
& +\left(\frac{\mu_{1} R_{2}}{16 \omega_{n}^{2}}-\frac{\mu_{1} R_{2}}{8 \omega_{m} \omega_{n}}\right)\left(p_{m}^{2}-q_{m}^{2}\right) \\
& \dot{q}_{n}=-\frac{\mu_{1}}{2} q_{n}+\left(2 \alpha-\delta+\frac{\mu_{1}^{2}}{8 \omega_{n}}\right) p_{n}+\frac{R_{2} F_{\Omega_{s}}}{4 \omega_{m}^{2} \omega_{n}} p_{m}+\frac{F_{2 \Omega_{s}}}{\omega_{n}} \\
& +\left(\frac{\mu_{1} R_{2}}{8 \omega_{n}^{2}}-\frac{\mu_{1} R_{2}}{4 \omega_{m} \omega_{n}}\right) p_{m} q_{m}+\frac{R_{2}}{4 \omega_{n}}\left(p_{m}^{2}-q_{m}^{2}\right) \\
& +\left(\frac{K_{3}}{8 \omega_{n}}+\frac{R_{1} R_{2}}{16 \omega_{m}^{2} \omega_{n}}\right)\left(q_{m}^{2} p_{n}+p_{m}^{2} p_{n}\right)+\frac{K_{4}}{8 \omega_{n}}\left(p_{n} q_{n}^{2}+p_{n}^{3}\right)
\end{aligned}
$$


B.2 Cartesian form of the modulation equations pertaining to three-to-one internal resonance.

$$
\begin{aligned}
\omega_{m} p_{m}{ }^{\prime}= & -\alpha \omega_{m} q_{m}-R_{2} q_{m} p_{n}{ }^{2}+2 R_{3} p_{m} q_{m} p_{n}-R_{3} p_{m}{ }^{2} q_{n}+R_{3} q_{m}{ }^{2} q_{n} \\
& -R_{2} q_{m} q_{n}{ }^{2}-R_{1} p_{m}{ }^{2} q_{m}-\mu_{1} \omega_{m} p_{m}-R_{1} q_{m}{ }^{3} \\
\omega_{m} q_{m}{ }^{\prime}= & \alpha \omega_{m} p_{m}+\bar{F}_{\Omega_{s}}+R_{2} p_{m} q_{n}{ }^{2}+2 R_{3} p_{m} q_{m} q_{n}-R_{3} q_{m}{ }^{2} p_{n} \\
& +R_{3} p_{m}{ }^{2} p_{n}+R_{2} p_{m} p_{n}{ }^{2}+R_{1} p_{m} q_{m}{ }^{2}+R_{1} p_{m}{ }^{3}-\mu_{1} \omega_{m} q_{m} \\
\omega_{m} p_{n}^{\prime}= & -3 \alpha \omega_{m} q_{n}-\delta \omega_{m} q_{n}+K_{1} p_{m}{ }^{2} q_{n}-K_{1} q_{m}{ }^{2} q_{n}-3 K_{3} p_{m}{ }^{2} q_{m} \\
& +K_{3} q_{m}{ }^{3}-K_{1} p_{n}{ }^{2} q_{n}-K_{1} q_{n}{ }^{3}-\mu_{2} \omega_{n} p_{n} \\
\omega_{m} q_{n}{ }^{\prime}= & 3 \alpha \omega_{m} p_{n}-\delta \omega_{m} p_{n}+K_{1} q_{m}{ }^{2} p_{n}+K_{1} p_{m}{ }^{2} p_{n}-3 K_{3} p_{m} q_{m}{ }^{2} \\
& +K_{3} p_{m}{ }^{3}+K_{1} p_{n} q_{n}{ }^{2}+K_{1} p_{n}{ }^{3}-\mu_{2} \omega_{n} q_{n}+\bar{F}_{3 \Omega_{s}}
\end{aligned}
$$

\section{Appendix C}

C.1 Frequency response curves of the cases plotted in Fig. 3 and Fig. 4 for two-to-one internal resonance

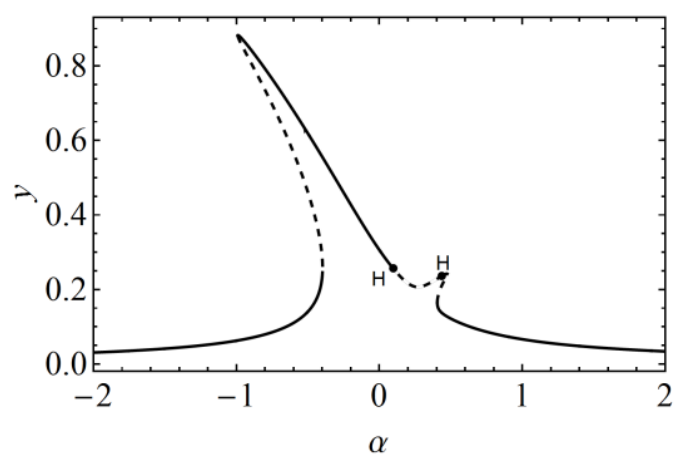

(a)

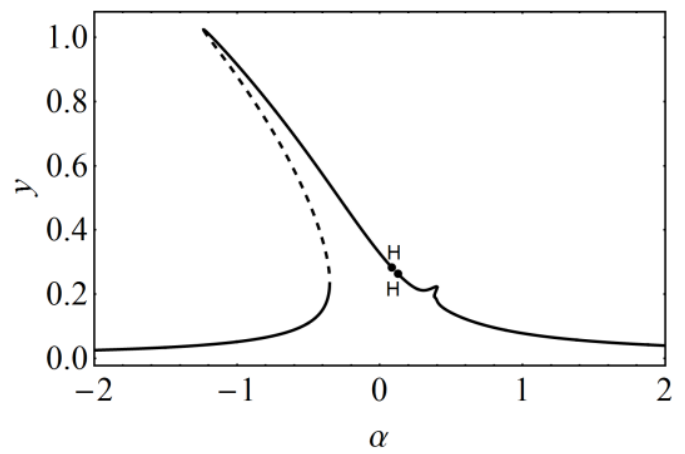

(c)

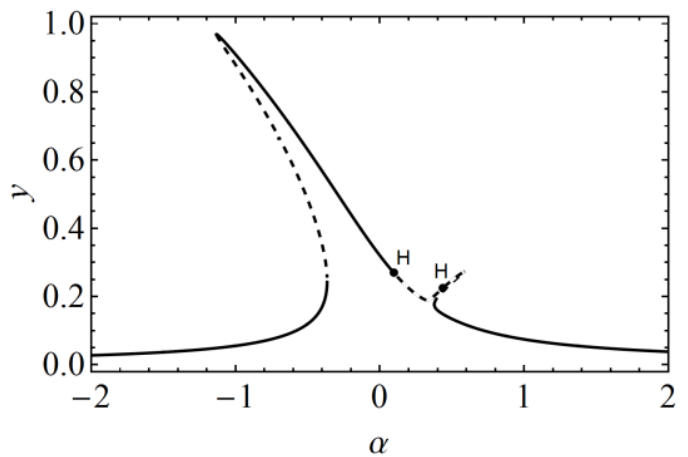

(b)

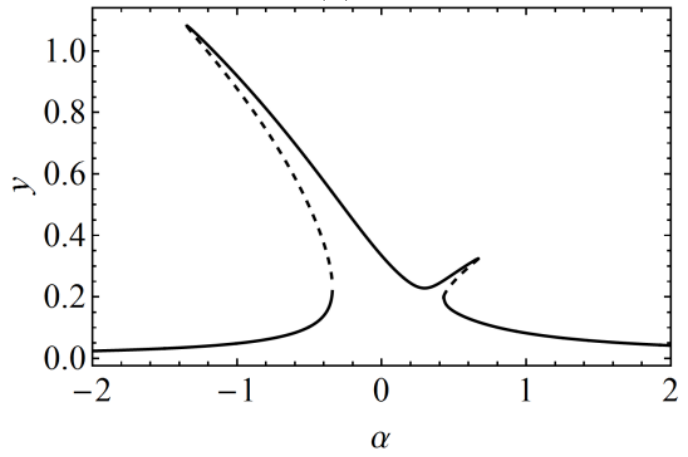

(d)

Figure C.1: Frequency response curve of the riser deflection as varying the detuning parameter $\alpha$. $\theta=62^{\circ}, \mu_{1}=0.08, \mu_{2}=0.08, F_{\Omega_{s}}=8$. (a) $F_{2 \Omega_{s}}=2$, (b) $F_{2 \Omega_{s}}=6$, (c) $F_{2 \Omega_{s}}=8$, (d) $F_{2 \Omega_{s}}=10$.

(• ) H denotes Hopf bifurcation. (-) Stable solution, (- - -) unstable solution. 
C.2 Frequency response curves of the cases plotted in Fig. 11 and Fig.

12 for three-to-one internal resonance

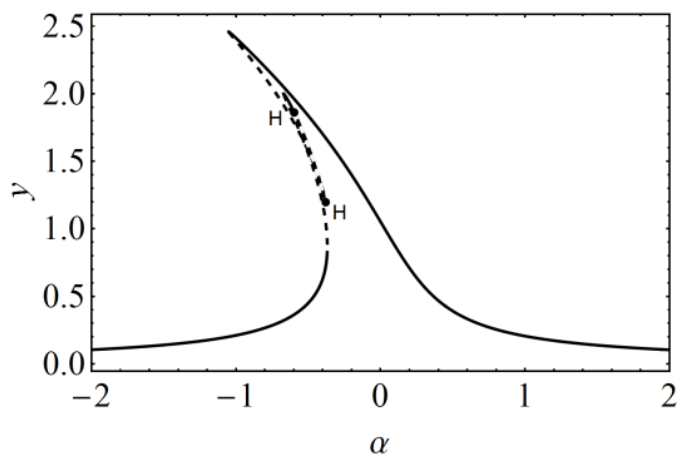

(a)

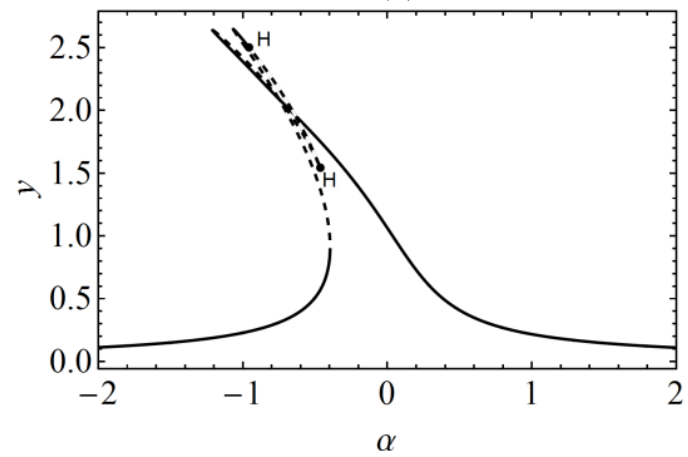

(c)

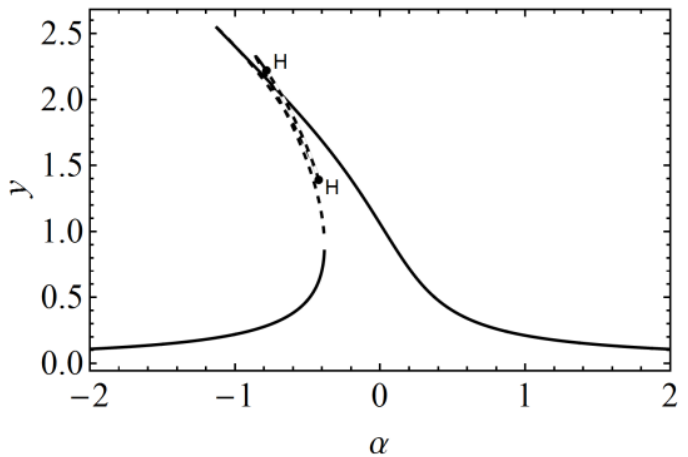

(b)

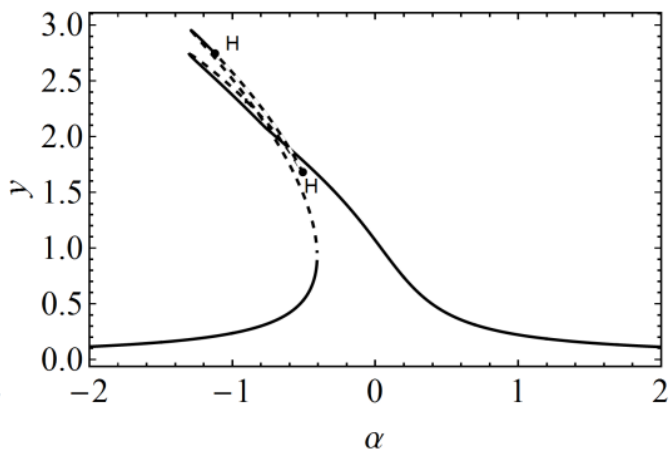

(d)

Figure C.2: Frequency response curve of the total deflection as varying the detuning parameter $\alpha$. $\theta=15^{\circ}, \mu_{1}=0.08, \mu_{2}=0.08, F_{\Omega_{s}}=169$. (a) $F_{3 \Omega_{s}}=50$, (b) $F_{3 \Omega_{s}}=100$, (c) $F_{3 \Omega_{s}}=150$, (d) $F_{3 \Omega_{s}}=200$.(• ) H denotes Hopf bifurcation. (—) Stable solution, (- - ) unstable solution.

\section{References}

1. Baarholm, G.S., Larsen, C.M., Lie, H.: On fatigue damage accumulation from in-line and cross-flow vortex-induced vibrations on risers. J. Fluids Struct. 22(1), 109-127 (2006).

2. Vandiver, J.K., Jaiswal, V., Jhingran, V.: Insights on vortex-induced, traveling waves on long risers. J. Fluids Struct. 25(4), 641-653 (2009). doi:http://dx.doi.org/10.1016/j.jfluidstructs.2008.11.005

3. Trim, A.D., Braaten, H., Lie, H., Tognarelli, M.A.: Experimental investigation of vortex-induced vibration of long marine risers. J. Fluids Struct. 21(3), 335-361 (2005). doi:http://dx.doi.org/10.1016/j.jfluidstructs.2005.07.014

4. Srinil, N., Ma, B., Zhang, L.: Experimental investigation on in-plane/out-ofplane vortex-induced vibrations of curved cylinder in parallel and perpendicular flows. J. Sound Vibr. 421, 275-299 (2018). doi:https://doi.org/10.1016/j.jsv.2018.02.021

5. Song, L., Fu, S., Cao, J., Ma, L., Wu, J.: An investigation into the hydrodynamics of a flexible riser undergoing vortex-induced vibration. J. Fluids Struct.

63 , $325-350$ (2016). doi:http://dx.doi.org/10.1016/j.jfluidstructs.2016.03.006 
6. Han, Q., Ma, Y., Xu, W., Lu, Y., Cheng, A.: Dynamic characteristics of an inclined flexible cylinder undergoing vortex-induced vibrations. J. Sound Vibr. 394, 306-320 (2017). doi:https://doi.org/10.1016/j.jsv.2017.01.034

7. Rateiro, F., Fujarra, A., Pesce, C., Gonçalves, R., Franzini, G.R., Mendes, P.: Experimental Investigations on Vortex-Induced Vibrations with a Long Flexible Cylinder. Part III: Modal-Amplitude Analysis with a Catenary Configuration. In: Proceedings of the 11th International Conference on Flow-Induced Vibration 2016

8. Nayfeh, A.: Quenching of primary resonance by a superharmonic resonance. J. Sound Vibr. 92(3), 363-377 (1984).

9. Nayfeh, A.: Quenching of a primary resonance by a combination resonance of the additive or difference type. J. Sound Vibr. 97(1), 65-73 (1984).

10. Plaut, R.H., HaQuang, N., Mook, D.T.: The influence of an internal resonance on non-linear structural vibrations under two-frequency excitation. J. Sound Vibr. 107(2), 309-319 (1986). doi:https://doi.org/10.1016/0022460X(86)90240-3

11. Plaut, R., Hsieh, J.-C.: Oscillations and instability of a shallow-arch under two-frequency excitation. J. Sound Vibr. 102(2), 189-201 (1985).

12. Pezeshki, C., Elgar, S., Krishna, R.C.: An examination of multi-frequency excitation of the buckled beam. J. Sound Vibr. 148(1), 1-9 (1991). doi:https://doi.org/10.1016/0022-460X(91)90817-4

13. Luongo, A., Piccardo, G.: Non-linear galloping of sagged cables in 1: 2 internal resonance. J. Sound Vibr. 214(5), 915-940 (1998).

14. Zhao, Y., Guo, Z., Huang, C., Chen, L., Li, S.: Analytical solutions for planar simultaneous resonances of suspended cables involving two external periodic excitations. Acta Mech. 229(11), 4393-4411 (2018).

15. Zhao, Y., Huang, C., Chen, L., Peng, J.: Nonlinear vibration behaviors of suspended cables under two-frequency excitation with temperature effects. J. Sound Vibr. 416, 279-294 (2018).

16. Nayfeh, A.H., Mook, D.T.: Nonlinear oscillations. John Wiley \& Sons, (2008)

17. Khalak, A., Williamson, C.H.K.: MOTIONS, FORCES AND MODE TRANSITIONS IN VORTEX-INDUCED VIBRATIONS AT LOW MASS-DAMPING. J. Fluids Struct. 13(7), 813-851 (1999). doi:https://doi.org/10.1006/jfls.1999.0236

18. Balasubramanian, S., Skop, R.A., Haan Jr, F.L., Szewczyk, A.A.: Vortexexcited vibrations of uniform pivoted cylinders in uniform and shear flow. $\begin{array}{llll}\text { J. Fluids } & \text { Struct. } & \text { 14(1), } & 65-85\end{array}$ doi:https://doi.org/10.1006/jfls.1999.0255

19. Facchinetti, M.L., de Langre, E., Biolley, F.: Coupling of structure and wake oscillators in vortex-induced vibrations. J. Fluids Struct. 19(2), 123-140 (2004). doi:http://dx.doi.org/10.1016/j.jfluidstructs.2003.12.004

20. Mazzilli, C.E., Sanches, C.T.: VORTEX-INDUCED INSTABILITIES IN OFFSHORE CATENARY RISERS: A NON-LINEAR NORMAL MODE APPROACH. In: 11th Pan-American Congress of Applied Mechanics, Brazil 2010

21. Srinil, N.: Multi-mode interactions in vortex-induced vibrations of flexible curved/straight structures with geometric nonlinearities. J. Fluids Struct. 26(7-8), $1098-1122$ (2010). doi:http://dx.doi.org/10.1016/j.jfluidstructs.2010.08.005 
22. Srinil, N., Wiercigroch, M., O'Brien, P.: Reduced-order modelling of vortexinduced vibration of catenary riser. Ocean Eng. 36(17-18), 1404-1414 (2009). doi:https://doi.org/10.1016/j.oceaneng.2009.08.010

23. Meng, D., Chen, L.: Nonlinear free vibrations and vortex-induced vibrations of fluid-conveying steel catenary riser. Appl. Ocean Res. 34(0), 52-67 (2012). doi:http://dx.doi.org/10.1016/j.apor.2011.10.002

24. Dai, H.L., Wang, L., Qian, Q., Ni, Q.: Vortex-induced vibrations of pipes conveying fluid in the subcritical and supercritical regimes. J. Fluids Struct.

39(0),

322-334

(2013). doi:http://dx.doi.org/10.1016/j.jfluidstructs.2013.02.015

25. Wu, J., Lie, H., Larsen, C.M., Liapis, S., Baarholm, R.: Vortex-induced vibration of a flexible cylinder: Interaction of the in-line and cross-flow $\begin{array}{llllll}\text { responses. J. Fluids } & \text { Struct. 63, 238-258 } & \text { (2016). }\end{array}$ doi:http://dx.doi.org/10.1016/j.jfluidstructs.2016.03.001

26. Wu, X., Ge, F., Hong, Y.: A review of recent studies on vortex-induced vibrations of long slender cylinders. J. Fluids Struct. 28, 292-308 (2012). doi:http://dx.doi.org/10.1016/j.jfluidstructs.2011.11.010

27. Alfosail, F.K., Younis, M.I.: Two-to-one internal resonance of an inclined marine riser under harmonic excitations. Nonlinear Dyn. (2018). doi:https://doi.org/10.1007/s11071-018-4630-2

28. Alfosail, F.K., Younis, M.I.: Three-to-one internal resonance of inclined marine riser. International Journal of Non-Linear Mechanics 109, 107-117 (2019). doi:https://doi.org/10.1016/j.ijnonlinmec.2018.11.008

29. Nayfeh, A.H., Owis, F., Hajj, M.R.: A Model for the Coupled Lift and Drag

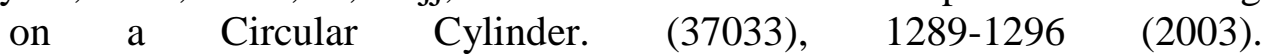
doi:10.1115/DETC2003/VIB-48455

30. Marzouk, O., Nayfeh, A.H., Akhtar, I., Arafat, H.N.: Modeling Steady-state and Transient Forces on a Cylinder. Journal of Vibration and Control 13(7), 1065-1091 (2007). doi:https://doi.org/10.1177/1077546307078737

31. Dahl, J.M., Hover, F.S., Triantafyllou, M.S., Oakley, O.H.: Dual resonance in vortex-induced vibrations at subcritical and supercritical Reynolds numbers. Journal of Fluid Mechanics 643, 395-424 (2010). doi:https://doi.org/10.1017/S0022112009992060

32. Alfosail, F.K., Nayfeh, A.H., Younis, M.I.: An analytic solution of the static problem of inclined risers conveying fluid. Meccanica 52(4), 1175-1187 (2016). doi:https://doi.org/10.1007/s11012-016-0459-2

33. Alfosail, F.K., Nayfeh, A.H., Younis, M.I.: Natural frequencies and mode shapes of statically deformed inclined risers. International Journal of Non$\begin{array}{llll}\text { Linear } & \text { Mechanics } & 94 & 12-19\end{array}$ doi:https://doi.org/10.1115/imece2016-66009

34. Paidoussis, M.: Dynamics of flexible slender cylinders in axial flow Part 1. Theory. Journal of Fluid Mechanics 26(04), 717-736 (1966).

35. Nayfeh, A.H.: Introduction to Perturbation Techniques. Wiley, (2011)

36. Nayfeh, A.H., Balachandran, B.: Applied nonlinear dynamics: analytical, computational and experimental methods. John Wiley \& Sons, (2008) 